\title{
A Variational Approach and Finite Element Implementation for Swelling of Polymeric Hydrogels under Geometric Constraints
}

\author{
Min Kyoo Kang and Rui Huang* \\ Department of Aerospace Engineering and Engineering Mechanics, University of Texas, Austin,
} $T X 78712$

\begin{abstract}
A hydrogel consists of a cross-linked polymer network and solvent molecules. Depending on its chemical and mechanical environment, the polymer network may undergo enormous volume change. The present work develops a general formulation based on a variational approach, which leads to a set of governing equations coupling mechanical and chemical equilibrium conditions along with proper boundary conditions. A specific material model is employed in a finite element implementation, for which the nonlinear constitutive behavior is derived from a free energy function, with explicit formula for the true stress and tangent modulus at the current state of deformation and chemical potential. Such implementation enables numerical simulations of hydrogels swelling under various constraints. Several examples are presented, with both homogeneous and inhomogeneous swelling deformation. In particular, the effect of geometric constraint is emphasized for inhomogeneous swelling of surface-attached hydrogel lines of rectangular cross-sections, which depends on the width-to-height aspect ratio of the line. The present numerical simulations show that, beyond a critical aspect ratio, crease-like surface instability occurs upon swelling.
\end{abstract}

Keywords: hydrogel, swelling, large deformation, surface instability

*ruihuang@mail.utexas.edu 


\section{Introduction}

An aggregate of a polymer network and small molecules (e.g., water) forms a polymeric hydrogel. In response to various environmental stimuli (e.g., temperature, vapor pressure, $\mathrm{pH}$, electric field), a hydrogel can swell or shrink dramatically by absorbing or desorbing the solvent molecules. The stimuli-responsive properties of hydrogels along with their degree of flexibility similar to natural tissues have led to a wide range of applications in biotechnology and medicine [1-4], including drug delivery, tissue engineering, biosensors, as well as other microdevices [5-7].

Complex material behaviors of hydrogels with large, reversible deformation and various instability patterns have been observed in experiments [8-17], which have motivated a large body of theoretical and numerical studies [18-30]. Recently, following the classical works by Gibbs [31] and Biot [32, 33], Hong et al. [27] formulated a nonlinear field theory coupling diffusion of solvent molecules and large deformation of polymeric gels. With a specific material model, such a theory enables analyses of swelling induced deformation phenomena in hydrogels under various physical and geometrical constraints [28-30].

In an effort to study the effects of constraint on swelling of polymeric thin films and nanolines [4, 9-17], the present paper reformulates the theory by Hong et al. [30] in a general variational form and develops an alternative method for finite element analysis of equilibrium states of polymeric hydrogels swollen under constraints. The remainder of this paper is organized as follows. Section 2 presents the general statements of the variational principle and derives the equilibrium equations along with boundary conditions for the coupled mechanical and chemical fields. Section 3 develops a finite element method for numerical simulations using the userdefined material (UMAT) feature of the commercial package ABAQUS. Analytical and 
numerical solutions are presented in Section 4 for homogeneous and inhomogeneous swelling of hydrogels, where the effects of geometric constraints are emphasized.

\section{Theory - A Variational Approach}

\subsection{General statements}

Consider a hydrogel body (current state) of volume $\Omega$ enclosed by a surface $\Gamma$, subjected to a distributed body force, $b_{i}$, and surface traction, $t_{i}$. In addition, the hydrogel is immersed in a solvent environment of chemical potential $\hat{\mu}$ (per solvent molecule), and transport of the solvent molecules occurs within the hydrogel body and across the surface $\Gamma$. As illustrated in Fig. 1, part of the surface $\Gamma$ may be mechanically constrained (e.g., attached to a rigid surface) and/or chemically isolated from the solvent.

With an infinitesimal variation to the current state in terms of both mechanical displacement and molecular transport, the total work done to the hydrogel includes the mechanical work by the body force and the surface traction and the chemical work via absorption of solvent molecules, namely,

$$
\delta W=\int_{\Omega} b_{i} \delta x_{i} d V+\oint_{\Gamma} t_{i} \delta x_{i} d S-\oint_{\Gamma} \hat{\mu} n_{k} \delta i_{k} d S,
$$

where $\delta x_{i}$ is the variation of the current position and $\delta i_{k}$ is the variation of the molecular flux, defined as the number of solvent molecules across per unit area of a surface element with the surface normal in the direction $x_{k}$. The vector product $-n_{k} \delta i_{k}$ gives the number of solvent molecules entering the gel across per unit area of its surface, where $n_{k}$ is the unit normal vector on the surface (positive outwards). We ignore the injection of solvent molecules by distributed pump that was included in the theory by Hong et al. [27]. Additional terms may be added in Eq. 
(1) to include works done by other fields (e.g., temperature, electric field), which is beyond the scope of the present study.

Assuming a free energy density function for the hydrogel, $u$, the variation of the total free energy of the hydrogel is

$$
\delta \Phi=\delta\left(\int_{\Omega} u d V\right)
$$

The functional form of $u$ determines the constitutive behavior of the hydrogel, which will be discussed later with a specific material model.

The variation of the free energy for the thermodynamic system including the hydrogel and its mechanical/chemical environment is

$$
\delta G=\delta \Phi-\delta W
$$

For all thermodynamically permissible variations, $\delta G \leq 0$ [34]. If the current state is a thermodynamically equilibrium state, $\delta G=0$ for any arbitrary variation. Otherwise, the system evolves to reduce its free energy $(\delta G<0)$.

Furthermore, mass conservation of the solvent molecules requires that

$$
\delta\left(\int_{\Omega} c d V\right)=-\oint_{\Gamma} n_{k} \delta i_{k} d S .
$$

where $c$ is the concentration of the solvent molecule in the hydrogel (i.e., number of molecules per unit volume at the current state). Eq. (4) simply states that the total number of solvent molecules in the gel changes only as the molecules enter or leave the gel through its boundary $(\Gamma)$, assuming no sources or distributed pumps inside the body $(\Omega)$. We emphasize that this statement does not assume incompressibility of the solvent molecules or the polymer network.

The left-hand side of Eq. (4) can be decomposed into two parts, namely

$$
\delta\left(\int_{\Omega} c d V\right)=\int_{\Omega} \delta c d V+\int_{\Omega} c \delta x_{k, k} d V,
$$


where the second term on the right-hand side of Eq. (5) represents the contribution from the volume change of the gel, with $\delta x_{k, k}$ being the linear volumetric strain for an infinitesimal variation from the current state.

By substituting Eq. (5) into Eq. (4) and applying the divergence theorem on the righthand side, we obtain that

$$
\int_{\Omega}\left(\delta c+c \delta x_{k, k}\right) d V=-\int_{\Omega} \delta i_{k, k} d V
$$

For Eq. (6) to hold everywhere inside the gel, it necessarily requires that

$$
\delta c=-\delta i_{k, k}-c \delta x_{k, k} \quad \text { in } \Omega
$$

Therefore, the general statements of the variational principle for the hydrogel include one for the variation of free energy (Eq. 3) and one for mass conservation (Eq. 4 or 7).

\subsection{Nominal quantities}

It is often convenient to use nominal quantities referring to a reference configuration with fixed volume $\Omega_{0}$ and surface $\Gamma_{0}$. As illustrated in Fig. 1, a deformation gradient tensor $\mathbf{F}$ maps the reference configuration to the current state, namely,

$$
d x_{i}=F_{i K} d X_{K} \text { and } F_{i K}=\frac{\partial x_{i}}{\partial X_{K}}
$$

where $X_{K}$ refers to the fixed coordinates at the reference state. While the choice of the reference state is arbitrary in general, we choose the dry state of the hydrogel as the reference state in the present study. As will be discussed later, such a choice is necessary for the use of a specific free energy function. On the other hand, it poses a numerical challenge that has to be circumvented in finite element analysis. 
The differential volume and surface area at the current state are related to those in the reference state by

$$
d V=J d V_{0} \text { and } n_{i} d S=A_{i J} N_{J} d S_{0}
$$

where $N_{J}$ is the unit normal of the surface at the reference state, and

$$
J=\operatorname{det}(\mathbf{F}), A_{i J}=\frac{1}{2} e_{i j k} e_{J K L} F_{j K} F_{k L}
$$

Thus, the nominal quantities (in upper cases) can be defined as follows:

- $\quad$ Nominal body force $\mathbf{B}: B_{i} d V_{0}=b_{i} d V$;

- $\quad$ Nominal surface traction T: $T_{i} d S_{0}=t_{i} d S$;

- $\quad$ Nominal molecular flux I: $N_{K} \delta I_{K} d S_{0}=n_{k} \delta i_{k} d S$;

- $\quad$ Nominal free energy density $U: U d V_{0}=u d V$;

- Nominal molecular concentration $C: C d V_{0}=c d V$.

In terms of the nominal quantities, the variational statements in Eqs. (1), (2), and (4) are recast as

$$
\begin{aligned}
& \delta W=\int_{\Omega_{0}} B_{i} \delta x_{i} d V_{0}+\oint_{\Gamma_{0}} T_{i} \delta x_{i} d S_{0}-\oint_{\Gamma_{0}} \hat{\mu} N_{K} \delta I_{K} d S_{0}, \\
& \delta \Phi=\int_{\Omega_{0}} \delta U d V_{0} \\
& \int_{\Omega_{0}} \delta C d V_{0}=-\oint_{\Gamma_{0}} N_{K} \delta I_{K} d S_{0} .
\end{aligned}
$$

Applying the divergence theorem to Eq. (13) leads to

$$
\delta C=-\frac{\partial}{\partial X_{K}}\left(\delta I_{K}\right) \quad \text { in } \Omega_{0} .
$$




\subsection{Equilibrium equations}

At the equilibrium state, $\delta G=\delta \Phi-\delta W=0$, and thus

$$
\int_{\Omega_{0}} \delta U d V_{0}=\int_{\Omega_{0}} B_{i} \delta x_{i} d V_{0}+\oint_{\Gamma_{0}} T_{i} \delta x_{i} d S_{0}-\oint_{\Gamma_{0}} \hat{\mu} N_{K} \delta I_{K} d S_{0}
$$

Assume a general form of the nominal free energy density function, $U(\mathbf{F}, C)$. Variation of the free energy at the left hand side of Eq. (15) can be carried out as follows:

$$
\begin{aligned}
\int_{\Omega_{0}} \delta U d V_{0} & =\int_{\Omega_{0}} \frac{\partial U}{\partial F_{i K}} \delta F_{i K} d V_{0}+\int_{\Omega_{0}} \frac{\partial U}{\partial C} \delta C d V_{0} \\
& =\int_{\Omega_{0}} \frac{\partial U}{\partial F_{i K}} \frac{\partial}{\partial X_{K}}\left(\delta x_{i}\right) d V_{0}-\int_{\Omega_{0}} \frac{\partial U}{\partial C} \frac{\partial}{\partial X_{K}}\left(\delta I_{K}\right) d V_{0}
\end{aligned}
$$

By applying the divergence theorem, we obtain that

$$
\begin{aligned}
\int_{\Omega_{0}} \delta U d V_{0}= & \oint_{\Gamma_{0}} \frac{\partial U}{\partial F_{i K}} N_{K} \delta x_{i} d S_{0}-\int_{\Omega_{0}} \frac{\partial}{\partial X_{K}}\left(\frac{\partial U}{\partial F_{i K}}\right) \delta x_{i} d V_{0} \\
& -\oint_{\Gamma_{0}} \frac{\partial U}{\partial C} N_{K} \delta I_{K} d S_{0}+\int_{\Omega_{0}} \frac{\partial}{\partial X_{K}}\left(\frac{\partial U}{\partial C}\right) \delta I_{K} d V_{0}
\end{aligned}
$$

Thus, the equilibrium condition in Eq. (15) becomes

$$
\begin{aligned}
& \oint_{\Gamma_{0}}\left(\frac{\partial U}{\partial F_{i K}} N_{K}-T_{i}\right) \delta x_{i} d S_{0}-\int_{\Omega_{0}}\left[\frac{\partial}{\partial X_{K}}\left(\frac{\partial U}{\partial F_{i K}}\right)+B_{i}\right] \delta x_{i} d V_{0} \\
& -\oint_{\Gamma_{0}}\left(\frac{\partial U}{\partial C}-\hat{\mu}\right) N_{K} \delta I_{K} d S_{0}+\int_{\Omega_{0}} \frac{\partial}{\partial X_{K}}\left(\frac{\partial U}{\partial C}\right) \delta I_{K} d V_{0}=0
\end{aligned}
$$

For Eq. (18) to hold for any arbitrary variations, it necessarily requires that

$$
\left\{\begin{array}{l}
\frac{\partial}{\partial X_{K}}\left(\frac{\partial U}{\partial F_{i K}}\right)+B_{i}=0 \\
\frac{\partial}{\partial X_{K}}\left(\frac{\partial U}{\partial C}\right)=0
\end{array}\right.
$$

in $\Omega_{0}$,

and 


$$
\begin{cases}\frac{\partial U}{\partial F_{i K}} N_{K}=T_{i} \text { or } \delta x_{i}=0 & \text { on } \Gamma_{0} . \\ \frac{\partial U}{\partial C}=\hat{\mu} \text { or } N_{K} \delta I_{K}=0 & \end{cases}
$$

The governing equations for the equilibrium state of the hydrogel are thus established in Eq. (19), along with the boundary conditions in Eq. (20). It is noted that, in the variational analysis, the deformation gradient $(\mathbf{F})$ and the concentration $(C)$ have been taken as the state variables in the definition of the free energy function $(U)$, while the mechanical displacement of the polymer network $\left(\delta x_{i}\right)$ and molecular flux of the solvent $\left(\delta I_{K}\right)$ are the physical processes that change the current state of the hydrogel. At the equilibrium state, the free energy $G$ is minimized with respect to any arbitrary variations in both the displacement and flux.

Now we may define the nominal stress and chemical potential as work conjugates of the deformation gradient and solvent concentration, respectively:

$$
s_{i K}=\frac{\partial U}{\partial F_{i K}} \text { and } \mu=\frac{\partial U}{\partial C} .
$$

The equilibrium equations and the boundary conditions are then re-written as

$$
\begin{cases}\frac{\partial s_{i K}}{\partial X_{K}}+B_{i}=0 & \text { in } \Omega_{0} \\ \frac{\partial \mu}{\partial X_{K}}=0 & \end{cases}
$$

and

$$
\left\{\begin{array}{l}
s_{i K} N_{K}=T_{i} \text { or } \delta x_{i}=0 \\
\mu=\hat{\mu} \text { or } N_{K} \delta I_{K}=0
\end{array} \quad \text { on } \Gamma_{0} .\right.
$$

We note that, in addition to the familiar boundary conditions for the mechanical traction (natural) and displacement (essential), the chemical boundary condition can be specified either 
by the chemical potential of the external solvent or by zero flux (e.g., surface isolated or blocked from the solvent). It is also possible to have mixed boundary conditions.

The two field equations in (22) appear to be uncoupled. However, both the nominal stress $s_{i K}$ and the chemical potential $\mu$ are derived from the same free energy density function $U$, which are coupled in general through the constitutive behavior of the hydrogel. The second equation (chemical equilibrium) dictates that the chemical potential be a constant at the equilibrium state (if it exists). This is only possible when the hydrogel is in contact with a homogeneous solvent of a constant chemical potential, i.e., $\mu=\hat{\mu}=$ const. The constant chemical potential in the hydrogel as an equilibrium condition is analogous to the constant temperature as an equilibrium condition for heat transfer.

The chemical potential of the external solvent, either in a liquid or a gaseous state, is given by [27]

$$
\hat{\mu}=\left\{\begin{array}{cc}
\left(p-p_{0}\right) v, & \text { if } p>p_{0} ; \\
k_{B} T \log \left(p / p_{0}\right), & \text { if } p<p_{0},
\end{array}\right.
$$

where $p$ is the pressure in the solvent, $p_{0}$ is the equilibrium vapor pressure, $v$ is the volume per solvent molecule, $T$ is the absolute temperature, and $k_{B}$ is the Boltzmann constant. At the equilibrium vapor pressure $\left(p=p_{0}\right)$, the external chemical potential $\hat{\mu}=0$.

At a non-equilibrium state, the solvent molecules migrate within the gel and the polymer network deforms to reduce the potential energy $G$, i.e., $\delta G<0$. Assuming self diffusion as the dominant kinetic process, Hong et al. [27] developed a kinetic model, based on which a finite element method was developed for transient analysis of swelling polymeric gels [30]. The present study focuses on analysis of equilibrium states only. 


\subsection{A free energy function for hydrogel}

In addition to the governing equations, a specific functional form of the free energy density, $U(\mathbf{F}, C)$, is needed for the analysis of swelling deformation of hydrogels. Following the approach by Flory [35], we adopt a free energy function that consists of two parts, one for elastic deformation of the polymer network and the other for mixing of the solvent molecules with the polymer chains, namely

$$
U(\mathbf{F}, C)=U_{e}(\mathbf{F})+U_{m}(C) .
$$

Based on a statistical mechanics model of rubber elasticity, the elastic free energy density was obtained by Flory $[35,36]$ as

$$
U_{e}(\mathbf{F})=\frac{1}{2} N k_{B} T\left[\lambda_{1}^{2}+\lambda_{2}^{2}+\lambda_{3}^{2}-3-\ln \left(\lambda_{1} \lambda_{2} \lambda_{3}\right)\right],
$$

where $\lambda_{1}, \lambda_{2}$, and $\lambda_{3}$ are the principal stretches in the principal directions of the deformation gradient tensor $\mathbf{F}$, and $N$ is the effective number of polymer chains per unit volume of the hydrogel at the dry state which is related to the cross-link density of the polymer network. It is well known that $N k_{B} T$ defines the initial shear modulus of an elastomer [37]. When the deformation does not change volume (i.e., $\lambda_{1} \lambda_{2} \lambda_{3}=1$ ), Eq. (26) reduces to the familiar strain energy density function for incompressible neo-Hookean materials [38]. For swelling deformation of a hydrogel, however, the volume changes dramatically. The last term in the bracket of Eq. (26), resulting from the entropy change associated with the volume change, is however problematic from a mechanics consideration [37, 39]. To account for the volume change in rubber elasticity, many other forms of the free energy function have been suggested [37, 39-42]. In the present study, following Hong et al. [27], we take the elastic free energy function as 


$$
U_{e}(\mathbf{F})=\frac{1}{2} N k_{B} T\left[\lambda_{1}^{2}+\lambda_{2}^{2}+\lambda_{3}^{2}-3-2 \ln \left(\lambda_{1} \lambda_{2} \lambda_{3}\right)\right]=\frac{1}{2} N k_{B} T\left(F_{i K} F_{i K}-3-2 \ln J\right),
$$

which differs from Eq. (26) by a factor of 2 in the volumetric term. The same functional form was suggested by others from mathematical considerations [39] as well as a statistical mechanics model $[43,44]$. Note that the principal stretches and deformation gradient in Eq. (27) are defined with respect to the dry state as the reference, which is assumed to be isotropic. The functional form should be modified accordingly if a different reference state is used.

Based on Flory-Huggins polymer solution theory $[35,45]$, the free energy change due to mixing of pure solvent with a polymer network was obtained as:

$$
\Delta F_{m}=k_{B} T\left[n_{1} \ln \varphi+\chi n_{1}(1-\varphi)\right],
$$

where $n_{1}$ is the number of solvent molecules, $\varphi$ is the volume fraction of the solvent, and $\chi$ is a dimensionless quantity that characterizes the interaction energy between the solvent and the polymer. The first term on the right hand side of Eq. (28) comes from the entropy of mixing, and the second term from the heat of mixing (enthalpy).

By the assumption of molecular incompressibility, the volume swelling ratio of the hydrogel is

$$
J=\frac{V}{V_{0}}=1+v C
$$

It then follows that $n_{1}=V_{0} C$ and $\varphi=\frac{v C}{1+v C}$. Thus, the free energy of mixing per unit volume is

$$
U_{m}(C)=\frac{\Delta F_{m}}{V_{0}}=\frac{k_{B} T}{v}\left(v C \ln \frac{v C}{1+v C}+\frac{\chi v C}{1+v C}\right) .
$$

Eq. (30) differs slightly from that given in Hong et al. [27] by a constant, which is insignificant for swelling deformation. At the dry state, we have $C=0$ and $U_{m}=0$. The tendency to increase the entropy of mixing (thus to decrease the free energy) drives the solvent molecules 
to enter the polymer network. This tendency to mix may be either opposed $(\chi>0)$ or enhanced $(\chi$ $<0$ ) by the heat of mixing, depending on the sign of $\chi$. Furthermore, as the process of absorption proceeds, the elastic energy of the network increases as a penalty of swelling. Ultimately, a state of equilibrium swelling may be obtained, in which the total free energy reaches a minimum.

In search for the equilibrium swelling state, the condition of molecular incompressibility in Eq. (29) can be imposed as a constraint that relates the solvent concentration $C$ to the deformation of the polymer network. In the cases of homogeneous swelling, a term with a Lagrange multiplier for the constraint can be added to the free energy function, namely

$$
U(\mathbf{F}, C)=U_{e}(\mathbf{F})+U_{m}(C)+\Pi(1+v C-J) .
$$

As defined in Eq. (21), the principal nominal stresses are obtained as

$$
\begin{aligned}
& s_{1}=\frac{\partial U}{\partial \lambda_{1}}=N k_{B} T\left(\lambda_{1}-\frac{1}{\lambda_{1}}\right)-\Pi \lambda_{2} \lambda_{3}, \\
& s_{2}=\frac{\partial U}{\partial \lambda_{2}}=N k_{B} T\left(\lambda_{2}-\frac{1}{\lambda_{2}}\right)-\Pi \lambda_{1} \lambda_{3}, \\
& s_{3}=\frac{\partial U}{\partial \lambda_{3}}=N k_{B} T\left(\lambda_{3}-\frac{1}{\lambda_{3}}\right)-\Pi \lambda_{1} \lambda_{2} .
\end{aligned}
$$

In Eq. (32), the Lagrange multiplier $\Pi$ represents the osmotic pressure, resulting from the condition of molecular incompressibility. For free, isotropic swelling, we have $s_{1}=s_{2}=s_{3}=0$

and $\lambda_{1}=\lambda_{2}=\lambda_{3}=\lambda$, which leads to an osmotic pressure, $\Pi=N k_{B} T\left(\frac{1}{\lambda}-\frac{1}{\lambda^{3}}\right)$. As expected, the osmotic pressure is zero at the undeformed dry state $(\lambda=1)$. The predicted osmotic pressure at the dry state would not vanish if the elastic free energy function in Eq. (26) instead of Eq. (27) was used.

The chemical potential in the swollen hydrogel is obtained from Eq. (31) as 


$$
\mu=\frac{\partial U}{\partial C}=k_{B} T\left[\ln \frac{\nu C}{1+\nu C}+\frac{1}{1+\nu C}+\frac{\chi}{(1+\nu C)^{2}}+N v\left(\frac{1}{\lambda}-\frac{1}{\lambda^{3}}\right)\right]
$$

The last term in the bracket of Eq. (33) represents a modification to the chemical potential due to elastic reaction of the polymer network. A similar formula for the chemical potential was obtained by Flory [35], with a factor of 2 difference in the last term. The difference results from the different forms of the elastic free energy function in Eqs. (26) and (27).

By setting $\mu=\hat{\mu}$ as the external chemical potential defined in Eq. (24) and noting $v C=\lambda^{3}-1$ by molecular incompressibility, the isotropic, homogeneous equilibrium swelling ratio can be solved from Eq. (33) as

$$
\lambda=\lambda\left(\frac{\hat{\mu}}{k_{B} T} ; N v, \chi\right)
$$

where the two dimensionless quantities $(N v$ and $\chi)$ characterize the material system, with $N$ for the polymer network structure, $v$ for the solvent molecules, and $\chi$ for the solvent-polymer interaction. The effect of the external environment (e.g., temperature and vapor pressure) is accounted for in Eq. (34) via the normalized chemical potential. As an example, at the equilibrium vapor pressure, we have $\hat{\mu}=0$ and $\lambda=3.390$ for a hydrogel with $\chi=0.1$ and $v N=$ $10^{-3}$; the corresponding volume ratio of swelling is: $J=\lambda^{3}=38.96$.

It is noted that the first term in the bracket of Eq. (33) is unbounded at the dry state (when $C=0$ ). This is consistent with the definition of the external chemical potential in Eq. (24), which approaches negative infinity as the vapor pressure approaches zero (i.e., vacuum). However, the negative infinite chemical potential at the dry state poses a challenge for numerical simulations of swelling deformation under constraints from the dry state, as will be discussed in the subsequent sections. 


\section{Formulation of a Finite Element Method}

Simple, analytical solutions can be obtained for homogeneous swelling of hydrogels from the above theoretical framework [27]. For inhomogeneous swelling with complex geometric and physical constraints, however, numerical approaches are often necessary [27-30]. With the variational form of the present theory, a finite element method may be developed to solve the coupled field equations in Eq. (22). Alternatively, following Hong et al. [29], a Legendre transformation of the free energy density function leads to

$$
\hat{U}(\mathbf{F}, \mu)=U(\mathbf{F}, C)-\mu C,
$$

which can then be used to solve for the equilibrium swelling deformation with a prescribed chemical potential $\mu$. Since the chemical potential must be a constant at the equilibrium state $(\mu=\hat{\mu})$, a standard nonlinear finite element method for hyperelasticity [46] can be employed to solve for the equilibrium swelling deformation field $(\mathbf{F})$. The concentration field $(C)$, which is inhomogeneous in general, can then be obtained from the condition of molecular incompressibility, i.e., $C=(J-1) / v$.

Substituting Eqs. (27) and (30) into the free energy function in Eq. (25) and then into Eq. (35) and replacing $v C$ with $J-1$, we obtain that

$$
\hat{U}(\mathbf{F}, \mu)=\frac{1}{2} N k_{B} T(I-3-2 \ln J)+\frac{k_{B} T}{v}\left[(J-1) \ln \frac{J-1}{J}+\chi \frac{J-1}{J}\right]-\frac{\mu}{v}(J-1),
$$

where $I=F_{i K} F_{i K}$. At the undeformed dry state, we have $J=1$ and $I=3$ so that $\hat{U}=0$. However, the chemical potential at the dry state is singular $(\mu=-\infty)$, which cannot be accurately specified for numerical simulations. To circumvent this inconvenience, an auxiliary configuration with a finite value of the chemical potential is used as the initial state in numerical simulations, as 
illustrated in Fig. 1. The choice of the initial state should be such that (a) the corresponding swelling deformation is homogeneous, and (b) the essential boundary conditions at the dry state are satisfied. The condition (a) allows the chemical potential at the initial state to be obtained analytically, and the condition (b) ensures that the effect of constraints on swelling by the essential boundary conditions is maintained at the initial state and throughout the subsequent swelling process. In a previous study [29], a free, isotropic swelling deformation is assumed for the initial state, which does not necessarily satisfy the condition (b) for swelling of hydrogels under geometric constraints. In the present study, we choose an initial state with swelling deformation in form of

$$
\mathbf{F}^{(\mathbf{1})}=\left[\begin{array}{ccc}
\lambda_{1}^{(1)} & 0 & 0 \\
0 & \lambda_{2}^{(1)} & 0 \\
0 & 0 & \lambda_{3}^{(1)}
\end{array}\right] .
$$

The three principal stretches at the initial state and the corresponding chemical potential $\left(\mu=\mu_{1}\right)$ depend on specific constraints imposed by the essential boundary conditions, as will be discussed in the next section.

As illustrated in Fig. 1, the total swelling deformation from the dry state is decomposed into two parts as:

$$
\mathbf{F}=\mathbf{F}^{(2)} \mathbf{F}^{(1)}
$$

where $\mathbf{F}^{(2)}$ is the deformation gradient from the initial state $\left(\mu=\mu_{1}\right)$ to the final state of equilibrium $(\mu=\hat{\mu})$ and is to be solved numerically by the finite element method.

The nonlinear constitutive behavior of a hydrogel can be specified as a user-defined material in a standard finite element package such as ABAQUS [47]. In particular, ABAQUS offers two options for such implementation, with the user subroutine UHYPER or UMAT. The 
former (UHYPER) is specialized for hyperelastic materials, but with the restriction that the initial state must be isotropic. Assuming isotropic swelling at the initial state, a user subroutine with UHYPER was developed previously [29]. In the present study, with a generally anisotropic initial state as given in Eq. (37), we develop an alternative implementation for swelling of hydrogels under constraints using a UMAT subroutine in ABAQUS. As a general material subroutine, the procedures for UMAT implementation are quite different from those for UHYPER. In the UHYPER subroutine, the free energy function and its derivatives with respect to the deviatoric strain invariants are coded [29]. The restriction of such an implementation to an isotropic initial state results from the decomposition of the deformation gradient into a volumetric part and a deviatoric part. The present implementation using UMAT removes this restriction, but requires lengthy derivation of explicit formula for the true (Cauchy) stress and its variation with respect to the current state in terms of a fourth-order tangent modulus tensor.

First, the nominal stress is obtained as

$$
s_{i K}=\frac{\partial \hat{U}}{\partial F_{i K}}=\frac{\partial \hat{U}}{\partial I} \frac{\partial I}{\partial F_{i K}}+\frac{\partial \hat{U}}{\partial J} \frac{\partial J}{\partial F_{i K}} .
$$

By definition, the Kirchoff stress is

$$
J \sigma_{i j}=s_{i K} F_{j K}=\left(\frac{\partial \hat{U}}{\partial I} \frac{\partial I}{\partial F_{i K}}+\frac{\partial \hat{U}}{\partial J} \frac{\partial J}{\partial F_{i K}}\right) F_{j K},
$$

where $\sigma_{i j}$ is the true stress at the current state. Using the free energy function in Eq. (36), we obtain that

$$
\begin{aligned}
& \frac{\partial \hat{U}}{\partial I}=\frac{1}{2} N k_{B} T \\
& \frac{\partial \hat{U}}{\partial J}=N k_{B} T\left[-\frac{1}{J}+\frac{1}{N v}\left(\ln \frac{J-1}{J}+\frac{1}{J}+\frac{\chi}{J^{2}}-\frac{\mu}{k_{B} T}\right)\right] .
\end{aligned}
$$


Furthermore, it can be shown that

$$
\frac{\partial I}{\partial F_{i K}}=2 F_{i K} \text { and } \frac{\partial J}{\partial F_{i K}}=\frac{1}{2} e_{i j k} e_{K Q R} F_{j Q} F_{k R} .
$$

Substituting Eqs. (41-43) into Eq. (40), we obtain an explicit formula for the true stress:

$$
\sigma_{i j}=N k_{B} T\left[J^{-\frac{1}{3}} \bar{B}_{i j}+\frac{\delta_{i j}}{N \nu}\left(\ln \frac{J-1}{J}+\frac{1-N v}{J}+\frac{\chi}{J^{2}}-\frac{\mu}{k_{B} T}\right)\right],
$$

where $\bar{B}_{i j}=J^{-2 / 3} F_{i K} F_{j K}$ is the deviatoric stretch tensor and $\delta_{i j}$ is the Kronecker delta.

Next, variation of the Kirchoff stress gives that

$$
\delta\left(J \sigma_{i j}\right)=N k_{B} T\left[\frac{2}{3} J^{-\frac{1}{3}} \bar{B}_{i j} \delta J+J^{\frac{2}{3}} \delta \bar{B}_{i j}+\frac{\delta_{i j}}{N \nu}\left(\ln \frac{J-1}{J}+\frac{1}{J-1}-\frac{\chi}{J^{2}}-\frac{\mu}{k_{B} T}\right) \delta J\right] .
$$

It can be shown that

$$
\begin{aligned}
& \delta J=J \delta D_{k k} \\
& \delta \bar{B}_{i j}=H_{i j k l}\left(\delta D_{k l}-\frac{\delta_{k l}}{3} \delta D_{m m}\right)+\bar{B}_{k j} \delta W_{i k}-\bar{B}_{i k} \delta W_{k j},
\end{aligned}
$$

where

$$
\begin{aligned}
& \delta D_{i j}=\frac{1}{2}\left(\delta L_{i j}+\delta L_{j i}\right), \\
& \delta W_{i j}=\frac{1}{2}\left(\delta L_{i j}-\delta L_{j i}\right), \\
& \delta L_{i j}=\frac{\partial}{\partial x_{j}}\left(\delta u_{i}\right), \\
& H_{i j k l}=\frac{1}{2}\left(\bar{B}_{j l} \delta_{i k}+\bar{B}_{i k} \delta_{j l}+\bar{B}_{j k} \delta_{i l}+\bar{B}_{i l} \delta_{j k}\right) .
\end{aligned}
$$


In the above, $\delta u_{i}$ is the variation displacement, $\delta D_{i j}$ is the symmetric part of the deformation gradient, and $\delta W_{i j}$ is the antisymmetric part (spin), all of which are variational quantities with respect to the current state.

By substituting Eqs. (46) and (47) into Eq. (45), we obtain that

$$
\delta\left(J \sigma_{i j}\right)=J C_{i j k} \delta D_{k l}+J\left(\sigma_{k j} \delta W_{i k}-\sigma_{i k} \delta W_{k j}\right),
$$

where an explicit formula for the tangent modulus tensor at the current state is obtained as

$$
C_{i j k l}=N k_{B} T\left[J^{-\frac{1}{3}} H_{i j k l}+\frac{1}{N \nu}\left(\ln \frac{J-1}{J}+\frac{1}{J-1}-\frac{\chi}{J^{2}}-\frac{\mu}{k_{B} T}\right) \delta_{i j} \delta_{k l}\right] .
$$

The second term on the right-hand side of Eq. (52) results from rotation of the local coordinates, which is not needed in the material subroutine [47]. The first term on the right-hand side of Eq. (53) gives the tangent modulus for an incompressible, neo-Hookean material.

With Eqs. (44) and (53) for the true stress and tangent modulus, a user subroutine is coded in the format of UMAT in ABAQUS. Following Hong et al. [29], the chemical potential is mimicked by a temperature-like quantity in the user subroutine, which is set to be a constant in the hydrogel at the equilibrium state. Analogous to thermally induced deformation, change of the chemical potential leads to swelling deformation of the hydrogel, and stress is induced if it is subject to any constraint. Several examples are presented in the next section for homogeneous and inhomogeneous swelling of hydrogels under constraints. For convenience, we normalize the key quantities as follows:

$$
\bar{U}=\frac{\hat{U}}{N k_{B} T}, \bar{\sigma}_{i j}=\frac{\sigma_{i j}}{N k_{B} T}, \bar{\mu}=\frac{\mu}{k_{B} T} .
$$




\section{Analytical Solutions and Numerical Examples}

In this section, we first consider three simple examples of homogeneous swelling of a hydrogel, one without constraint and two with constraint. Numerical results are compared to the corresponding analytical solutions as benchmarks for the finite element implementation. Next, inhomogeneous swelling of surface-attached hydrogel lines is considered to further emphasize the effect of geometric constraint.

\subsection{Free, isotropic swelling}

As discussed in Section 2, under no constraint a hydrogel swells isotropically, for which the equilibrium swelling ratio $\lambda$ can be solved analytically by setting the chemical potential, $\mu=\hat{\mu}$, in Eq. (33). Figure 2 plots the equilibrium swelling ratio as a function of the external chemical potential for a hydrogel with $\chi=0.1$ and $v N=10^{-3}$.

For numerical analysis by the finite element method, an isotropic initial state is used for this case, with an arbitrary swelling ratio, $\lambda_{1}^{(1)}=\lambda_{2}^{(1)}=\lambda_{3}^{(1)}=1.5$. The chemical potential at the initial state is calculated analytically from Eq. (33). Then, the chemical potential of the hydrogel is increased gradually as the loading parameter in the finite element analysis until $\mu=0$, and the swelling ratio is calculated at each increment. A single three-dimensional 8-node brick element is used to model the hydrogel, with all boundaries free of traction. The numerical results are compared to the analytical solution in Figure 2, showing excellent agreement. Since the initial state is isotropic in this case, both the UHYPER and UMAT subroutines in ABAQUS can be used, and they produce identical results. 


\subsection{Anisotropic, homogeneous swelling of a hydrogel thin film}

Next consider a hydrogel thin film bonded to a rigid substrate, which swells preferably in the thickness direction due to the constraint in the lateral direction. For a thin film with its thickness dimension much smaller than its lateral dimensions, the swelling deformation is homogeneous, but anisotropic. Let 1 and 3 be the in-plane coordinates and 2 the out-of-plane coordinate. Under the lateral constraint, the principal stretches of the hydrogel thin film are: $\lambda_{1}=\lambda_{3}=1$ and $\lambda_{2}>1$. The lateral constraint induces a biaxial compressive stress in the film, i.e., $s_{1}=s_{3}=s<0$, while the other principal stress is zero, i.e., $s_{2}=0$, as the top surface of the film is assumed to be traction free. The osmotic pressure in the hydrogel thin film is obtained from the second of Eq. (32) as

$$
\Pi=N k_{B} T\left(\lambda_{2}-\frac{1}{\lambda_{2}}\right),
$$

The chemical potential is then obtained from Eq. (31) as

$$
\mu=\frac{\partial U}{\partial C}=k_{B} T\left[\ln \left(1-\frac{1}{\lambda_{2}}\right)+\frac{1}{\lambda_{2}}+\frac{\chi}{\lambda_{2}^{2}}+N v\left(\lambda_{2}-\frac{1}{\lambda_{2}}\right)\right],
$$

where the condition of molecular incompressibility, $v C=\lambda_{2}-1$, has been incorporated. Thus, by setting $\mu=\hat{\mu}$ in Eq. (56), we can solve for the equilibrium swelling ratio $\lambda_{2}$ for the hydrogel film as a function of the external chemical potential. The swelling induced stress in the hydrogel film is then obtained from the first and third of Eq. (32) as

$$
S_{1}=S_{3}=s=-N k_{B} T\left(\lambda_{2}^{2}-1\right) .
$$

The analytical solutions for the swelling ratio and the true stress $\left(\sigma=s / \lambda_{2}\right)$ are plotted in Fig. 3 for a hydrogel film with $\chi=0.1$ and $N v=10^{-3}$. The equilibrium swelling ratio at $\mu=\hat{\mu}=0$ is $\lambda_{2}=7.696$. Compared to the isotropic, free swelling $(\lambda=3.390$ and 
$J=\lambda^{3}=38.96$ ), while the linear stretch in the thickness direction of the film is larger, the volume ratio of swelling is much smaller for the hydrogel film $\left(J=\lambda_{2}=7.696\right)$, as a result of the lateral constraint.

To apply the finite element method for the anisotropic swelling of a hydrogel film, an anisotropic initial state is used, with $\lambda_{1}^{(1)}=\lambda_{3}^{(1)}=1$ and $\lambda_{2}^{(1)}=1.5$. The chemical potential at the initial state is calculated analytically from Eq. (56). In addition, the swelling-induced stress at the initial state is obtained from Eq. (57) and specified by a user subroutine SIGNI in ABAQUS [47]. Either three-dimensional brick elements or two-dimensional plane-strain elements can be used to model the hydrogel film. The lateral constraint on swelling is enforced by the boundary conditions. The numerical results are compared to the analytical solutions in Fig. 3, with excellent agreements for both the swelling ratios and the induced stresses as the chemical potential increases.

A similar problem was considered by Hong et al. [29] using a UHYPER material subroutine. There, an isotropic initial state with $\lambda_{1}^{(1)}=\lambda_{2}^{(1)}=\lambda_{3}^{(1)}=1.5$ was used, which relaxed the effect of lateral constraint. The corresponding chemical potential at the isotropic initial state was obtained from eq. (33) instead of Eq. (56), and no initial stress was induced. While the subsequent swelling was constrained in the lateral directions, their results are different from the present ones, as shown in Fig. 3. In particular, with the use of an isotropic initial state, the results (both swelling ratio and induced stress) for the subsequent swelling under the lateral constraint would depend on the choice of the initial state, and the corresponding analytical solution is different from that in Eqs. (55)-(57). With the UMAT implementation and an anisotropic initial state, the present results are independent of the initial state. 
Experimental observations of the swelling behavior of hydrogel thin films have shown good agreements with the theoretical predictions $[4,9]$. However, at high degrees of swelling, the homogeneous deformation becomes unstable and gives way to inhomogeneous deformation in form of surface wrinkles or creases $[9,10,12,15,16]$. In the present study, a similar surface instability is observed in numerical simulations for inhomogeneous swelling of surface-attached hydrogel lines in Section 4.4.

\subsection{Anisotropic, homogeneous swelling of a hydrogel line}

As another example, we consider swelling of a hydrogel line. Assume that the longitudinal dimension of the line is much larger than its lateral dimensions. Swelling of such a long line is constrained in the longitudinal direction, and thus $\lambda_{3}=1$. On the other hand, swelling in the lateral directions is unconstrained and isotropic, with $\lambda_{1}=\lambda_{2}=\lambda>1$. Such a constrained swelling induces a compressive longitudinal stress in the line: $s_{3}<0$, whereas $s_{1}=s_{2}=0$. From the first and second of Eq. (32), the osmotic pressure in the hydrogel line is

$$
\Pi=N k_{B} T\left(1-\frac{1}{\lambda^{2}}\right) .
$$

The chemical potential in the hydrogel line is obtained from Eq. (31) as

$$
\mu=\frac{\partial U}{\partial C}=k_{B} T\left[\ln \left(1-\frac{1}{\lambda^{2}}\right)+\frac{1}{\lambda^{2}}+\frac{\chi}{\lambda^{4}}+N v\left(1-\frac{1}{\lambda^{2}}\right)\right],
$$

where the condition of molecular incompressibility, $v C=\lambda^{2}-1$, has been applied. Thus, by setting $\mu=\hat{\mu}$ in Eq. (59), we can solve for the equilibrium swelling ratio $\lambda$ in the lateral direction for the hydrogel line as a function of the external chemical potential. The swelling induced longitudinal stress in the hydrogel line is then obtained from the third of Eq. (32) as 


$$
s_{3}=-N k_{B} T\left(\lambda^{2}-1\right) .
$$

The analytical solutions for the swelling ratio and the true stress $\left(\sigma_{3}=s_{3} / \lambda^{2}\right)$ are plotted in Fig. 4 for a hydrogel line with $\chi=0.1$ and $N v=10^{-3}$. The equilibrium swelling ratio of the hydrogel line at $\mu=\hat{\mu}=0$ is $\lambda=4.573$, and the volume swelling ratio is $J=\lambda^{2}=20.92$. Since the longitudinal constraint (1D) in the hydrogel line is weaker than the lateral constraint (2D) in the hydrogel film, the volume ratio of the line is greater than that of the film $\left(J=\lambda_{2}=7.696\right)$, but still smaller than that of the unconstrained, isotropic swelling $\left(J=\lambda^{3}=38.96\right)$.

For numerical simulations, we assume an anisotropic initial state, with $\lambda_{3}^{(1)}=1$ and an arbitrary swelling ratio in the lateral directions, $\lambda_{1}^{(1)}=\lambda_{2}^{(1)}=1.5$. The chemical potential at the initial state is calculated analytically from Eq. (59), and the swelling-induced stress at the initial state, obtained from Eq. (60), is specified by a user subroutine SIGNI in ABAQUS. The longitudinal constraint on swelling of the line is conveniently imposed by the plane-strain condition in the finite element analysis using the two-dimensional 4-node plane-strain elements, with traction-free boundary conditions on the side faces. As shown in Fig. 4, the numerical results agree closely with the analytical solutions for both the swelling ratios and the longitudinal stresses, independent of the choice of the auxiliary initial state.

\subsection{Inhomogeneous swelling of surface-attached hydrogel lines}

In this section, we consider swelling of hydrogel lines bonded to a rigid substrate. Polymer lines of this type are commonly used in lithography and imprinting processes for micro/nano-fabrication $[13,48]$, where large swelling deformation can be detrimental. Similar to the previous section, the longitudinal dimension of the line is assumed to be much larger than its 
lateral dimensions so that swelling is constrained in the longitudinal direction with $\lambda_{3}=1$. In addition, the line has a rectangular cross section at the dry state, with one of the side faces bonded to the substrate, as shown in Fig. 5(a). The bonding imposes an additional constraint on the lateral swelling of the line, and the effect of the constraint varies with the width-to-height aspect ratio $(\mathrm{W} / H)$ of its cross section. Swelling deformation of such a surface-attached hydrogel line is typically inhomogeneous, which offers a model system for the study of the constraint effect between two homogeneous limits: (i) When $W / H \rightarrow \infty$, the swelling becomes homogenous, as discussed in Section 4.2 for a hydrogel thin film; (ii) When $W / H \rightarrow 0$, the lateral constraint by the substrate becomes negligible, and the swelling becomes homogeneous and laterally isotropic, as discussed in Section 4.3 for a unattached hydrogel line.

Except for the two limiting cases, no analytical solution is available for inhomogeneous swelling of the surface-attached hydrogel lines. To apply the finite element method, we start from an anisotropic initial state of homogeneous swelling with $\lambda_{1}^{(1)}=\lambda_{3}^{(1)}=1$ and an arbitrarily selected swelling ratio in the height direction of the line, e.g., $\lambda_{2}^{(1)}=2$ as shown in Fig. 5(b). Such an initial state is identical to that for homogeneous swelling of a hydrogel thin film in Section 4.2, for which the chemical potential $\left(\mu=\mu_{1}\right)$ can be analytically calculated from Eq. (56). With $\lambda_{1}^{(1)}=\lambda_{3}^{(1)}=1$, the longitudinal constraint is maintained and the essential boundary condition at the bottom face of the line is satisfied at the initial state. However, the lateral constraint $\left(\lambda_{1}^{(1)}=1\right)$ imposes a compressive stress (or pressure $p$ ) onto the side faces of the line, as given in Eq. (57), which apparently violates the traction-free (natural) boundary condition of the intended problem. To recover the traction-free condition on the side faces of the line, we gradually release the imposed side pressure in Fig. 5(b) during the first step of numerical simulation, while keeping the chemical potential in the hydrogel unchanged. As illustrated in Fig. 5(c), the release of the 
side pressure leads to an inhomogeneous deformation of the hydrogel line at the initial chemical potential $\left(\mu=\mu_{1}\right)$. Subsequently, further swelling of the hydrogel line is simulated by gradually increasing the chemical potential until $\mu=0$, as shown in Fig. 5(d). We emphasize that the current implementation requires a homogeneously swollen initial state, while the mechanical boundary conditions may be controlled to facilitate the implementation. Due to the singularity in the chemical potential at the dry state, direct simulation from Fig. 5(a) to Fig. 5(d) is numerically intractable.

In all simulations of the present study, the dimensionless material parameters, $N v$ and $\chi$, are set to be 0.001 and 0.1 , respectively. The dry-state width-to-height aspect ratio $(W / H)$ is varied between 0.1 and 12. A relatively fine finite-element mesh is required for simulating inhomogeneous swelling deformation, especially at locations such as the lower corners where a high strain gradient is expected. The use of two-dimensional plane-strain elements is thus warranted by both the computational efficiency and the longitudinal constraint $\left(\lambda_{3}=1\right)$. For each model, the finite element mesh is refined until the result converges satisfactorily. The bonding of the bottom face of the hydrogel line to the rigid substrate is mimicked by applying a zerodisplacement (essential) boundary condition; debonding of the line is possible but not considered in the present study. Furthermore, the large deformation due to swelling often results in contact of the side faces of the hydrogel line with the substrate surface, for which hard and frictionless contact properties are assumed in the numerical simulations.

Figure 6(a) plots the average longitudinal stress as a function of the chemical potential for two hydrogel lines with $W / H=1$ and 10 . The analytical solutions at the two limiting cases are also plotted as the upper and lower bounds. At the initial state, we have $\lambda_{2}^{(1)}=1.2$ and the corresponding chemical potential, $\bar{\mu}_{1}=-0.8886$. The initial longitudinal stress is identical to that 
in a hydrogel film ( $W / H \rightarrow \infty)$, which can be obtained from Eq. (57) and lies on the solid line in Fig. 6(a). Upon release of the side pressure at the initial state, the magnitude of the average longitudinal stress is reduced while the chemical potential remains at the initial value. From the same initial state, the reduced stress magnitudes are different for the two hydrogel lines, higher in the line with $W / H=10$ than that in the line with $W / H=1$, due to stronger constraint in the line with the larger aspect ratio. Subsequently, as the chemical potential increases, the magnitudes of the average longitudinal stress in both the hydrogel lines increase. All the numerical results lie between the two homogeneous limits, while the stress magnitude increases with the aspect ratio $W / H$ at the same chemical potential.

Figure 6(b) plots the volume ratios of swelling for the two hydrogel lines as the chemical potential approaches $\mu=0$. The volume ratios increase as the chemical potential increases. The difference in the volume ratios of the two lines is less appreciable until the chemical potential is close to zero. Again, the two analytical limits set the upper and lower bounds for the volume swelling ratios of the surface-attached hydrogel lines. The larger the aspect ratio $W / H$, the stronger the constraint effect and thus the smaller the volume ratio of swelling at the same chemical potential.

The inhomogeneous swelling deformation along with the distribution of the longitudinal stress at the equilibrium chemical potential $\mu=0$ is plotted in Fig. 7 for three hydrogel lines with $W / H=1,5$, and 10 . For each line, the cross section at the dry state is outlined by a small rectangular box. The large swelling deformation pushes the side faces of the hydrogel lines to form contact with the rigid substrate surface. The contact length increases as the aspect ratio increases, reaching a full contact of the side faces for the hydrogel line with $W / H=10$. The stress contours show stress concentration at the bottom corners, where debonding may occur. We note 
that the magnitude of the stress in Fig. 7 is normalized by $N k_{B} T$, which is typically in the range of $10^{4} \sim 10^{7} \mathrm{~Pa}$ for polymeric hydrogels at the room temperature.

To further illustrate the effect of geometric constraint on swelling, Figure 8 plots the equilibrium swelling ratio at $\mu=0$ as a function of the dry-state width-to-height aspect ratio $(W / H)$ of the hydrogel lines. The two analytical limits are plotted as dashed lines. As the aspect ratio decreases, the effect of constraint by the substrate diminishes, and the volume ratio approaches that for the homogeneous swelling of a hydrogel line without any lateral constraint (upper bound). On the other hand, as the aspect ratio increases, the volume ratio decreases due to increasing constraint by the substrate, approaching the other limit for the homogeneous swelling of a hydrogel film (lower bound). Therefore, the degree of swelling can be tuned between the two homogeneous limits by varying the geometric aspect ratio of the surface-attached hydrogel lines.

As the aspect ratio $W / H$ increases beyond 10, swelling deformation of the hydrogel line becomes highly constrained and induces an increasingly large compressive stress at the top surface. It is found that, at a critical aspect ratio, a surface instability develops, as shown in Fig. 9 for $W / H=12$. As the chemical potential increases, the top surface of the hydrogel line evolves from nearly flat to slightly undulated, and eventually forms two crease-like foldings with selfcontact. The stress contours show stress concentration at the tip of the creases. More creases are observed in the simulation for a hydrogel line with the aspect ratio $W / H=13$. However, the numerical simulation becomes increasingly unstable with formation of the surface creases, posing a numerical challenge for simulations of hydrogel lines with higher aspect ratios. It is also noted that the contact of the side faces of the hydrogel line with the substrate surface plays an important role giving rise to the compressive stresses in the hydrogel. In simulations without 
enforcing the contact, the hydrogel line swelled more significantly and wrapped around the bottom surface until penetration or self contact, while surface creases were not observed.

Formation of surface creases has been observed experimentally in swelling gels $[9,10,12$, $16]$ as well as in rubbers under mechanical compression [49, 50]. A linear perturbation analysis by Biot [51] showed that homogeneous deformation of a rubber under compression becomes unstable at a critical strain, which is about 0.46 under plane-strain compression and about 0.33 under equi-biaxial compression. However, the theoretical prediction for the plane-strain compression was found to exceed the experimentally determined critical strain $(\sim 0.35)$ for rubbers [49]. In a recent experimental study of surface-attached hydrogel thin films [16], an effective linear compressive strain of $\sim 0.33$ was obtained for the onset of creasing in laterally constrained hydrogels. While this effective critical strain is remarkably close to Biot's prediction for rubbers under equi-biaxial compression, the critical condition for the onset of swell-induced creasing in hydrogels has not been established theoretically. A few recent efforts are noted [52, 53]. The present study of the surface-attached hydrogel lines offers an alternative approach. Typically for theoretical and numerical studies of surface instability, it is necessary to introduce perturbations to the reference homogeneous solution to trigger the instability. In the present study, surface creases form automatically in the numerical simulations for hydrogel lines beyond the critical aspect ratio, without any perturbation. Our numerical simulations show that the critical aspect ratio for the onset of surface instability depends on the external chemical potential and the material parameters of the hydrogel, i.e., $(W / H)_{c}=f(\bar{\mu}, N v, \chi)$. Therefore, the critical condition for surface instability in a laterally constrained hydrogel film $(W / H \rightarrow \infty)$ can be expressed in terms of the same parameters: if $f(\bar{\mu}, N v, \chi)<\infty$, the film surface is unstable; otherwise, the film surface is stable. A detailed stability analysis will be presented elsewhere. 


\section{Summary}

We have formulated a general variational approach for equilibrium analysis of swelling deformation of hydrogels. The governing equations for mechanical and chemical equilibrium are obtained along with the boundary conditions. A specific material model is adopted based on a free energy density function. A finite element method for numerical analysis is developed, which allows anisotropic initial states for the study of swelling of hydrogels under constraints. Numerical results by the finite element method are compared to analytical solutions for homogeneous swelling of hydrogels, both without and with constraint. The close agreements demonstrate the robustness of the present approach. Inhomogeneous swelling of hydrogel lines attached to a rigid substrate is simulated, illustrating the effect of geometric constraint with different width-to-height aspect ratios. Of particular interest is the formation of swelling-induced surface creases in the hydrogel lines beyond a critical aspect ratio. The present theoretical and numerical method can be used to study the complex swelling behavior of polymeric hydrogels under various geometric constraints, including buckling and creasing instabilities as observed in experiments [9-17].

\section{ACKNOWLEDGMENTS}

The authors gratefully acknowledge the financial support by National Science Foundation through Grant No. CMMI-0654105. Discussions with Dr. Wei Hong of Iowa State University have been helpful. 


\section{References}

1. Galaev, I. Y., Mattiasson, B., 1999, "'Smart' polymers and what they could do in biotechnology and medicine", Trends in Biotechnology, 17 (8), pp. 335-340.

2. Peppas, N.A., Hilt, J.Z., Khademhosseini, A., Langer, R., 2006, "Hydrogels in biology and medicine: From molecular principles to bionanotechnology”, Advanced Materials, 18 (11), pp. 1345-1360.

3. Uljin, R. V., Bibi, N., Jayawarna, V., Thornton, P. D., Todd, S. J., Mart, R. J., Smith A. M., Gough, J. E., 2007, "Bioresponsive hydrogels", Materials Today, 10 (4), pp. 40-48.

4. Tokarev, I, Minko, S., 2009, "Stimuli-responsive hydrogel thin films", Soft Matter, 5, pp. 511-524.

5. Beebe, D. J., Moore, J. S., Bauer, J. M., Yu, Q., Liu, R. H., Devadoss, C., Jo, B.-H., 2000, "Functional hydrogel structures for autonomous flow control inside microfluidic channels", Nature, 404, pp. 588-590.

6. Dong. L., Agarwal, A. K., Beebe., D. J., Jiang, H., 2006, “Adaptive liquid microlenses activated by stimuli-responsive hydrogels", Nature, 442, pp. 551-554.

7. Sidorenko, A., Krupenkin, T., Taylor, A., Fratzl, P. Aizenberg, J., 2007, "Reversible switching of hydrogel-actuated nanostructures into complex micropatterns', Science, 315, pp. 487-490.

8. Kuhn, W., Hargitay, B., Katchalsky, A., Eisenberg, H., 1950, "Reversible dilation and contraction by changing the state of ionization of high-polymer acid networks", Nature 165, pp. 514-516.

9. Southern, E., Thomas, A.G., 1965, "Effect of constraints on the equilibrium swelling of rubber vulcanizates”, J. Polymer Science A 3, pp. 641-646.

10. Tanaka, T., Sun, S.-T., Hirokawa, Y., Katayama, S., Kucera, J., Hirose, Y., Amiya, T., 1987, "Mechanical instability of gels at the phase transition", Nature, 325, pp. 796-198.

11. Matsuo, E. S., Tanaka, T., 1992, "Patterns in shrinking gels", Nature 358, pp. 482-485.

12. Tanaka, H., Sigehuzi, T., 1994, "Surface-pattern evolution in a swelling gel under a geometrical constraint: direction observation of fold structure and its coarsening dynamics", Phys. Rev. E 49, pp. R39-R42.

13. Tirumala, V. R., Divan, R., Ocola, L. E., Mancini, D. C., 2005, "Direct-write e-beam patterning of stimuli-responsive hydrogel nanostructures", J. Vac. Sci. Technol. B, 23(6), pp. 3124-3128.

14. Mora, T., Boudaoud, A., 2006, "Buckling of swelling gels", European Physical Journal E, 20 (2), pp. 119-124.

15. Sultan, E., Boudaoud, A., 2008, "The buckling of a swollen thin gel layer bound to a compliant substrate", Journal of Applied Mechanics, 75, 051002.

16. Trujillo, V., Kim, J., Hayward, R. C., 2008, "Creasing instability of surface-attached hydrogels", Soft Matter, 4, pp. 564-569. 
17. Zhang, Y., Matsumoto, E. A., Peter, A., Lin, P.-C., Kamien, R. D., Yang, S., 2008, "Onestep nanoscale assembly of complex structures via harnessing of an elastic instability", Nano Letters, 8, pp. 1192-1196.

18. Li, Y., Tanaka, T., 1992, "Phase transitions of gels", Annu. Rev. Mater. Sci., 22, pp. 243277.

19. Onuki, A., 1989, "Theory of pattern formation in gels: surface folding in highly compressible elastic bodies", Physical Review A 39 (11), pp. 5932-5948.

20. Suematsu, N., Sekimoto, N., Kawasaki, K., 1990, "Three-dimensional computer modeling for pattern formation on the surface of an expanding polymer gel", Physical Review A, 41 (10), pp. 5751-5754.

21. Durning, C. J., Morman, Jr., K. N., 1993, "Nonlinear swelling of polymer gels", J. Chemical Physics, 98 (5), pp. 4275-4293.

22. Wilder, J., Vilgis, T. A., 1998, "Elasticity in strongly interacting soft solids: A polyelectolyte network", Physical Review E, 57, pp. 6865-6874.

23. Maskawa, J., Takeuchi, T., Maki, K., Tsujii, K., Tanaka, T., 1999, “Theory and numerical calculation of pattern formation in shrinking gels", J. Chemical Physics 110 (22), pp. 1099310999.

24. Boudaoud, A., Chaieb, S., 2003, "Mechanical phase diagram of shrinking cylindrical gels", Phys. Rev. E 68, 021801.

25. Dolbow, J., Fried, E., Jia, H. D., 2004, “Chemically induced swelling of hydrogels", Journal of the Mechanics and Physics of Solids, 52, pp. 51-84.

26. Li, H., Luo, R., Birgersson, E., Lam, K. Y., 2007, "Modeling of multiphase smart hydrogels responding to $\mathrm{pH}$ and electric voltage coupled stimuli", J. Appl. Physics, 101 (11), 114905.

27. Hong, W., Zhao, X., Zhou, J., Suo, Z., 2008, "A theory of coupled diffusion and large deformation in polymeric gels", Journal of the Mechanics and Physics of Solids, 56, pp. 1779-1793.

28. Zhao, X, Hong, W. Suo, Z., 2008, "Stretching and polarizing a dielectric gel immersed in a solvent", International Journal of Solids and Structures, 45, pp. 4021-4031.

29. Hong, W., Liu, Z., and Suo, Z., 2009, "Inhomogeneous swelling of a gel in equilibrium with a solvent and mechanical load", International Journal of Solids and Structures 46, pp. 32823289.

30. Zhang, J., Zhao, X., Suo, Z., Jiang, H., 2009, “A finite element method for transient analysis of concurrent large deformation and mass transport in gels", Journal of Applied Physics, 105, 093522.

31. Gibbs, J. W., 1878, The scientific papers of J. Willard Gibbs, pp. 184, 201, 215.

32. Biot, M. A., 1941, "General theory of three-dimensional consolidation", Journal of Applied Physics, 12, pp. 155-164.

33. Biot, M.A., 1973, "Nonlinear and Semilinear Rheology of Porous Solids", J. Geophys. Res. 78, pp. 4924-4937. 
34. Prigogine, I., 1967, Introduction to Thermodynamics of Irreversible Processes ( $3^{\mathrm{rd}} \mathrm{ed}$ ), Wiley, New York.

35. Flory, P.J., 1953, Principles of Polymer Chemistry, Cornell University Press, Ithaca, NY.

36. Flory, P. J., 1950, "Statistical mechanics of swelling of network structures", Journal of Chemical Physics, 18, pp. 108-111.

37. Treloar, L.R.G., 1975, The Physics of Rubber Elasticity, Oxford University Press, Oxford.

38. Rivlin, R.S., 1948, "Large elastic deformations of isotropic materials. I. Fundamental concepts", Philosophical Trans. Royal Soc. London, A 240, pp. 459-490.

39. Simo, J. C., and Pister, K. S., 1984, "Remarks on rate constitutive equations for finite deformation problems: computational implications", Computer Methods in Applied Mechanics and Engineering, 46, pp. 201-215.

40. Ogden, R.W., 1976, "Volume changes associated with the deformation of rubber-like solids", J. Mech. Phys. Solids, 24 (6), pp. 323-338.

41. Bischoff, J.E., Arruda, E.M., Grosh, K., 2001, "A new constitutive model for the compressibility of elastomers at finite deformations", Rubber Chemistry and Technology, 74 (4), pp. 541-559.

42. Boyce, M.C., Arruda, E.M., 2000, "Constitutive models of rubber elasticity: A review", Rubber Chemistry and Technology, 73 (3), pp. 504-523.

43. Kuhn, W., Pasternak, R., Kuhn, H., 1947, "Mechanische und optische eigenschaften von gequollenem kautschuk", Helv. Chim Acta, 30, pp. 1705-1740.

44. Hermans, J. J., 1947, "Deformation and swelling of polymer networks containing comparatively long chains", Trans. Faraday Soc. 43, pp. 591-600.

45. Huggins, M.L., 1941, "Solutions of long chain compounds", J. Chem. Phys. 9 (5), pp. 440.

46. Belytschko, T., Liu, W.K., Moran, B., 2000, Nonlinear Finite Elements for Continua and Structures, John Wiley \& Sons.

47. ABAQUS (version 6.8), 2008, Dassault Systèmes Simulia Corp., Providence, RI, USA.

48. Hartschuh, R.D., Kisliuk, A., Novikov, V., Sokolov, A.P., Heyliger, P.R., Flannery, C.M., Johnson, W.L., Soles, C.L., Wu, W.-L., 2005, "Acoustic modes and elastic properties of polymeric nanostructures", Appl. Phys. Lett., 87, 173121.

49. Gent, A.N. and Cho, I.S., 1999, "Surface instabilities in compressed or bent rubber blocks", Rubber Chemistry and Technology 72, pp. 253-262.

50. Ghatak, A., Das, A.L., 2007, "Kink instability of a highly deformable elastic cylinder", Physical Review Letters 99, 076101.

51. Biot, M.A., 1963, "Surface instability of rubber in compression", Appl. Sci. Res. A 12, pp. 168-182.

52. Hohlfeld, E.B., 2008, "Creasing, Point-bifurcations, and Spontaneous Breakdown of Scaleinvariance", PhD Thesis, Harvard University, UMI Number 3312383.

53. Hong, W., Zhao, X., Suo, Z., 2009, "Formation of creases on the surface of elastomers and gels", submitted. 


\section{Figure Captions}

Figure 1. Schematic illustration of the reference state (dry) and the equilibrium state (swollen) of a hydrogel, along with an auxiliary initial state used in numerical simulations.

Figure 2. Comparison between numerical results and the analytical solution for free, isotropic swelling of a hydrogel.

Figure 3. Anisotropic swelling of a hydrogel film under lateral constraint: (a) the swelling ratio in the thickness direction; (b) swelling induced true stress in the lateral direction. Numerical results from two different implementations (UMAT and UHYPER) are compared to the analytical solution in Eqs. (56) and (57). Note that the results from UHYPER correspond to a different analytical solution with an isotropic initial swelling [29].

Figure 4. Anisotropic swelling of a hydrogel line under longitudinal constraint: (a) the swelling ratio in the lateral direction; (b) swelling induced true stress in the longitudinal direction.

Figure 5. Numerical steps to simulate inhomogeneous swelling of a hydrogel line $(W / H=1)$ attached to a rigid substrate: (a) the dry state; (b) the initial state; (c) deformation after releasing the side pressure in (b); (d) equilibrium swelling at $\mu=0$, with the dashed box as the scaled dry state.

Figure 6. Inhomogeneous swelling of surface-attached hydrogel lines: (a) average longitudinal stress; (b) volume ratio of swelling. The solid and dashed lines are analytical solutions for the homogeneous limits with $W / H \rightarrow \infty$ and $W / H \rightarrow 0$, respectively.

Figure 7. Simulated swelling deformation and longitudinal stress distribution in surface-attached hydrogel lines of different aspect ratios: (a) $W / H=1$; (b) $W / H=5$; (c) $W / H=10$. The rectangular boxes outline the cross sections at the dry state.

Figure 8. Equilibrium volume ratio as a function of the dry-state width-to-height aspect ratio for inhomogeneous swelling of surface-attached hydrogel lines.

Figure 9. Formation of surface creases in a surface-attached hydrogel line with $W / H=12$ as the chemical potential increases: (a) $\bar{\mu}=-0.00075$, (b) $\bar{\mu}=-0.0003$, and (c) $\bar{\mu}=0$. 


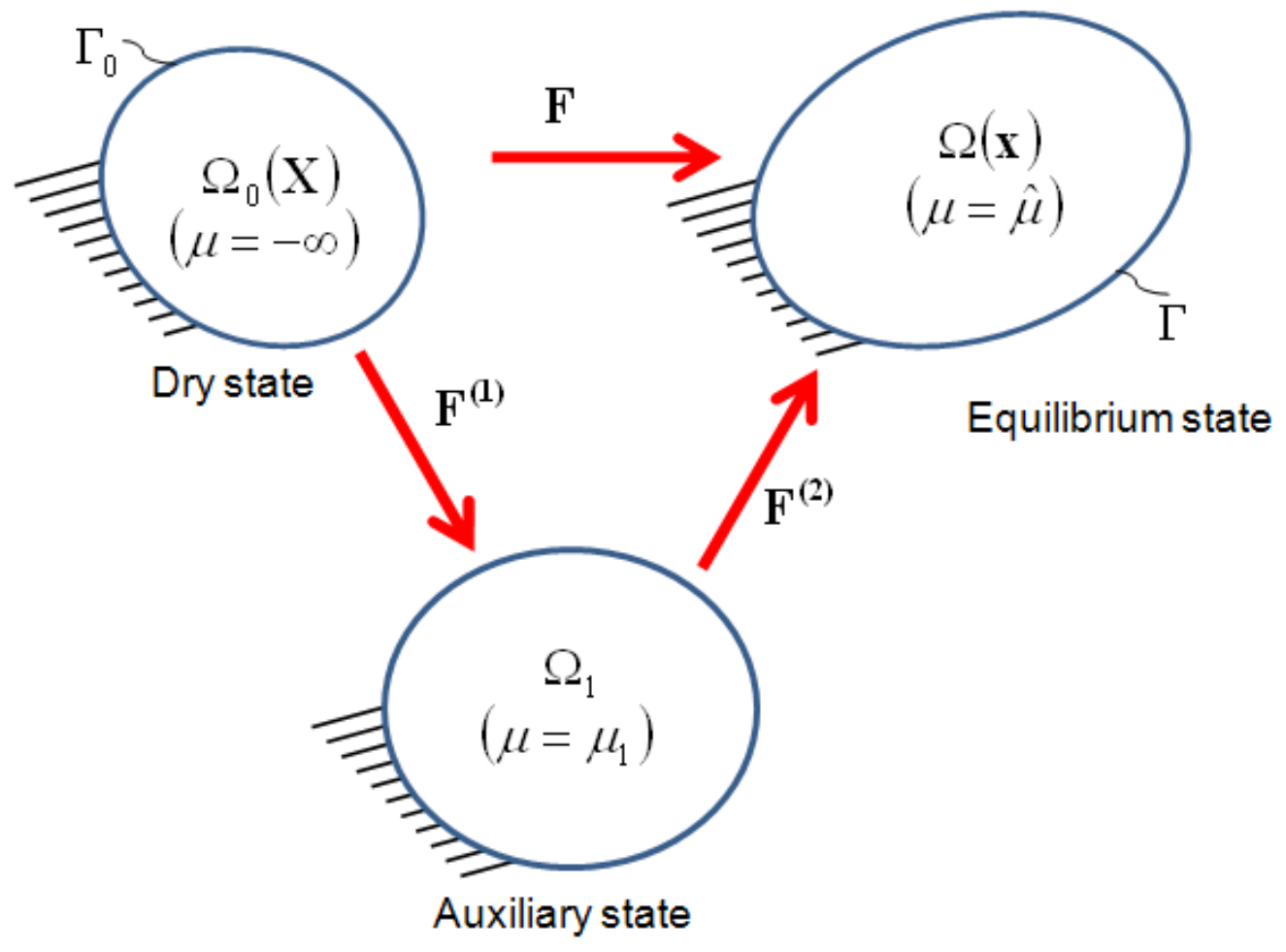

Figure 1. Schematic illustration of the reference state (dry) and the equilibrium state (swollen) of a hydrogel, along with an auxiliary initial state used in numerical simulations. 


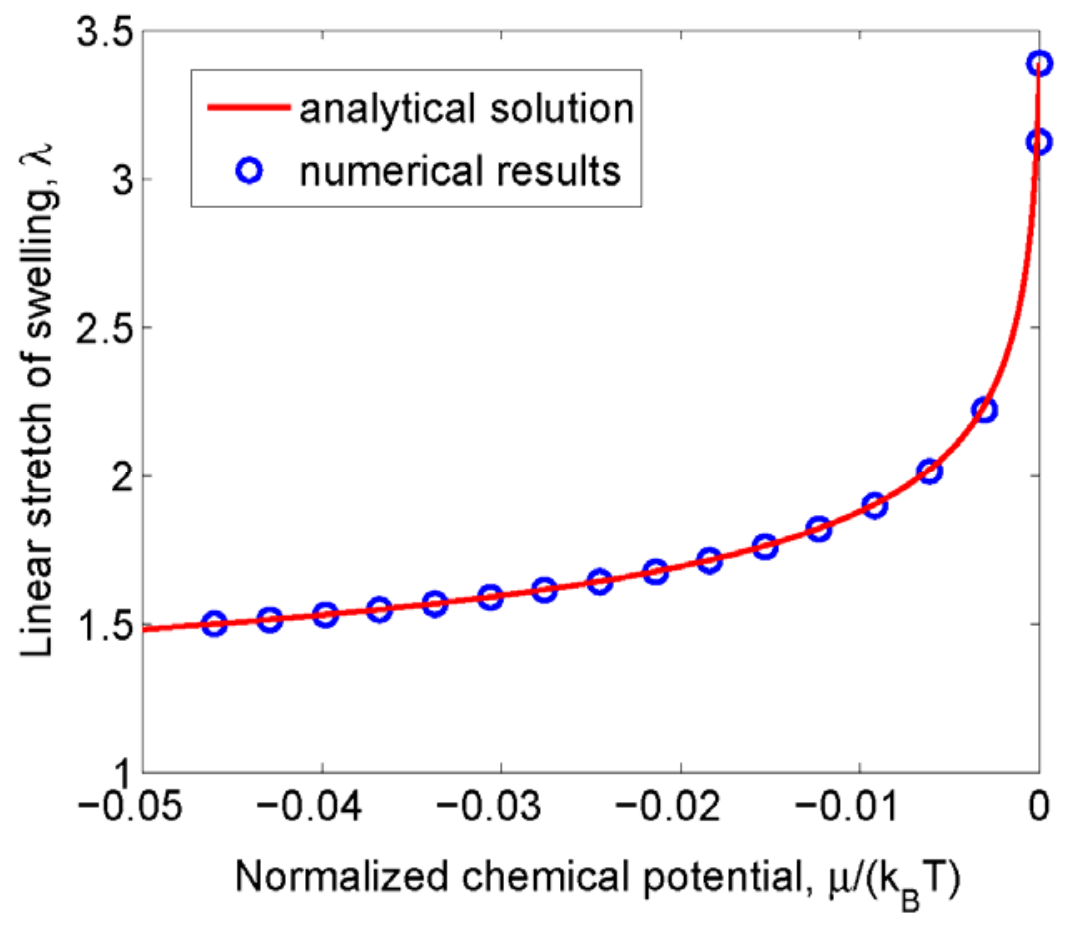

Figure 2. Comparison between numerical results and the analytical solution for free, isotropic swelling of a hydrogel. 


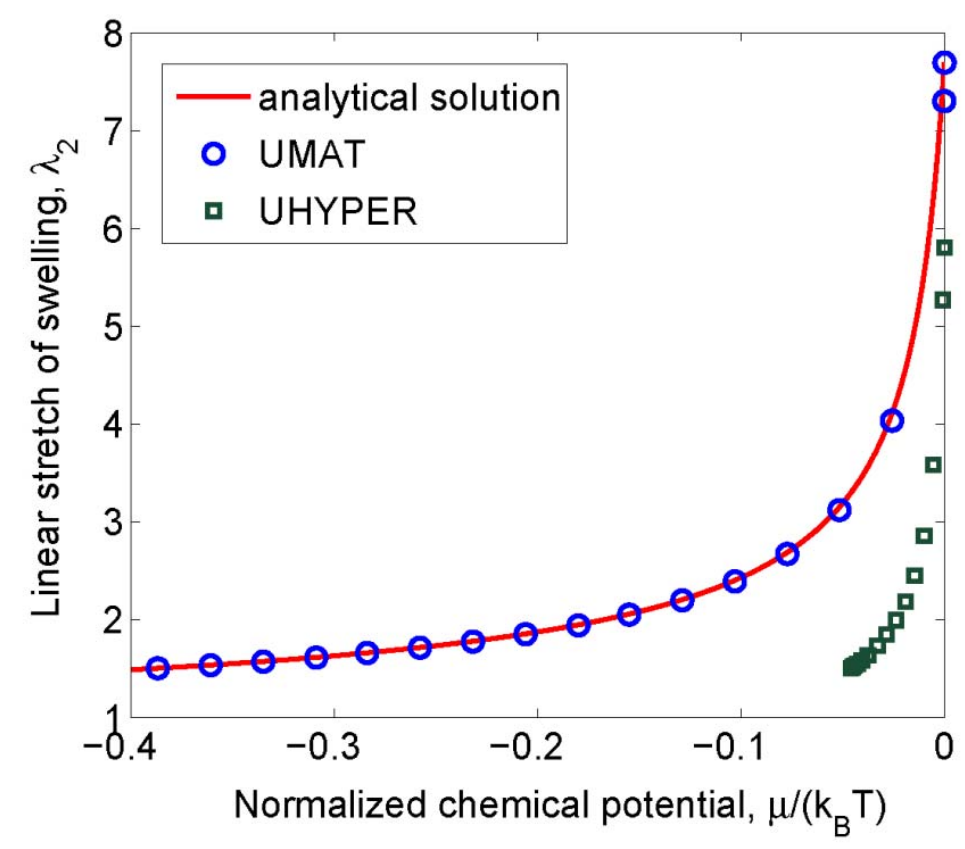

(a)

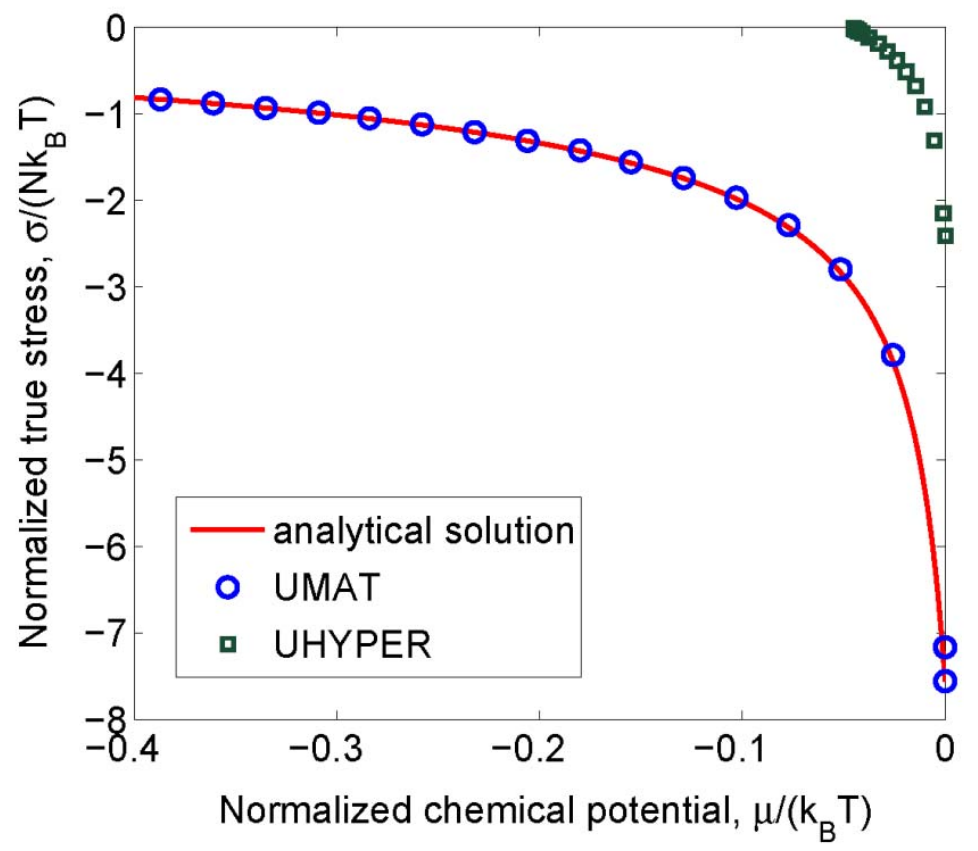

(b)

Figure 3. Anisotropic swelling of a hydrogel film under lateral constraint: (a) the swelling ratio in the thickness direction; (b) swelling induced true stress in the lateral direction. Numerical results from two different implementations (UMAT and UHYPER) are compared to the analytical solution in Eqs. (56) and (57). Note that the results from UHYPER correspond to a different analytical solution with an isotropic initial swelling [29]. 


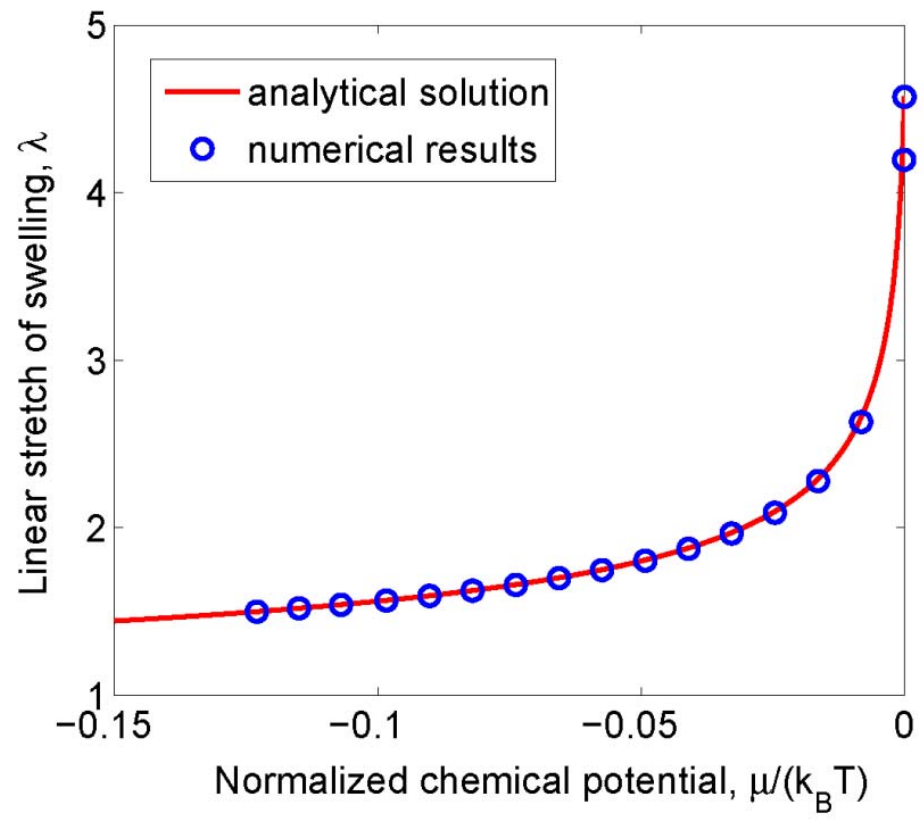

(a)

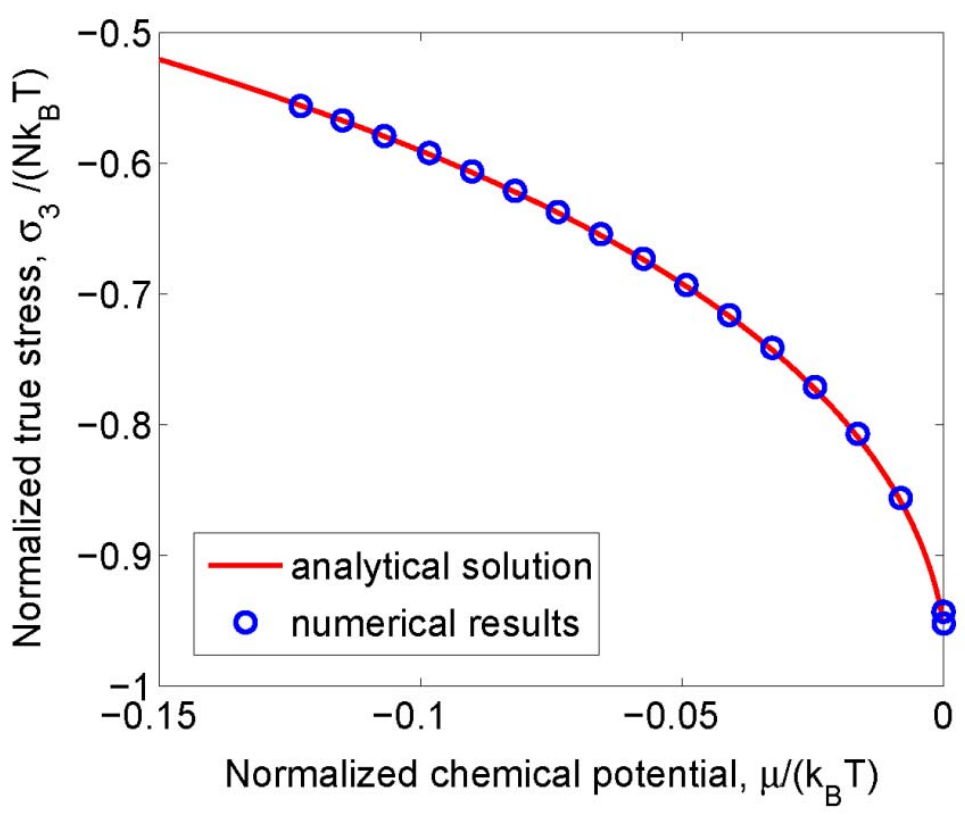

(b)

Figure 4. Anisotropic swelling of a hydrogel line under longitudinal constraint: (a) the swelling ratio in the lateral direction; (b) swelling induced true stress in the longitudinal direction. 


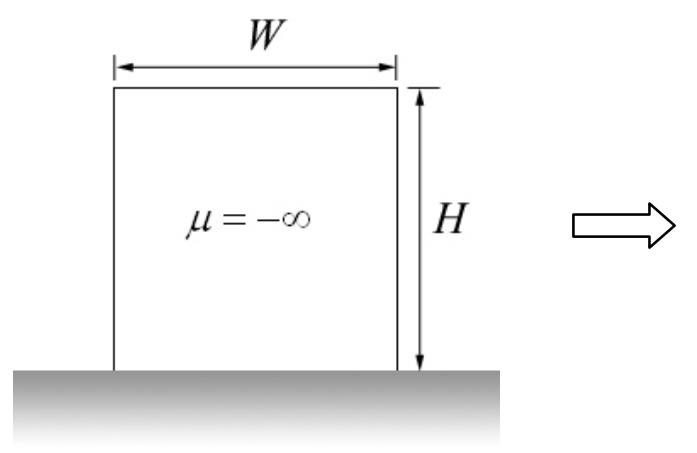

(a)

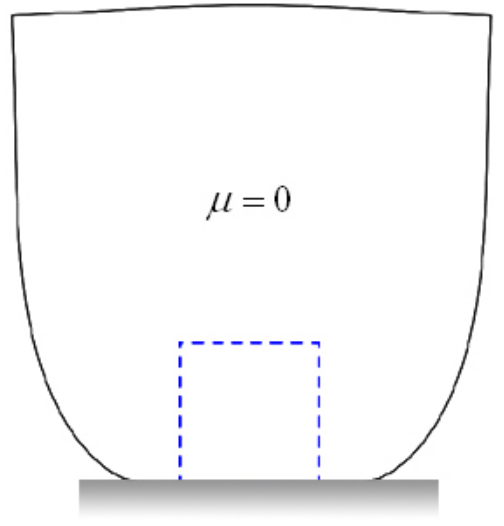

(d)

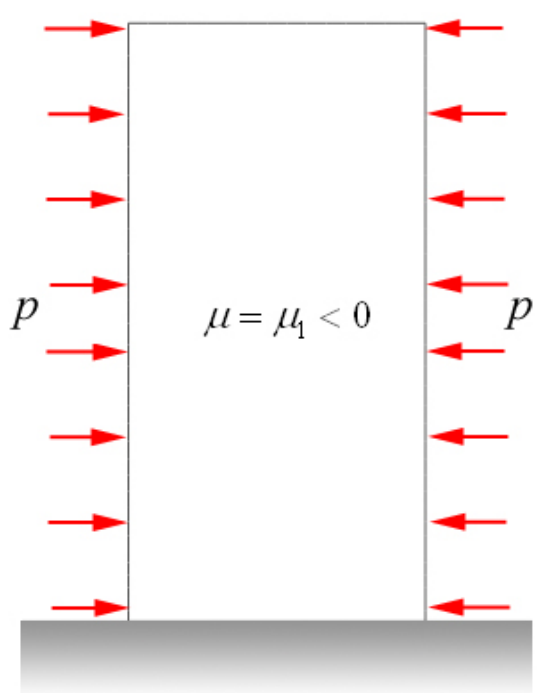

(b)
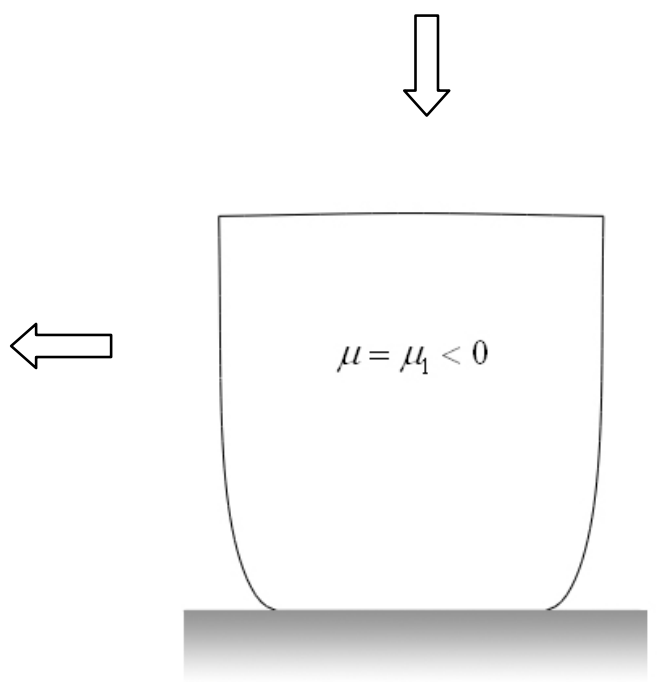

(c)

Figure 5. Numerical steps to simulate inhomogeneous swelling of a hydrogel line $(W / H=1)$ attached to a rigid substrate: (a) the dry state; (b) the initial state; (c) deformation after releasing the side pressure in (b); (d) equilibrium swelling at $\mu=0$, with the dashed box as the scaled dry state. 


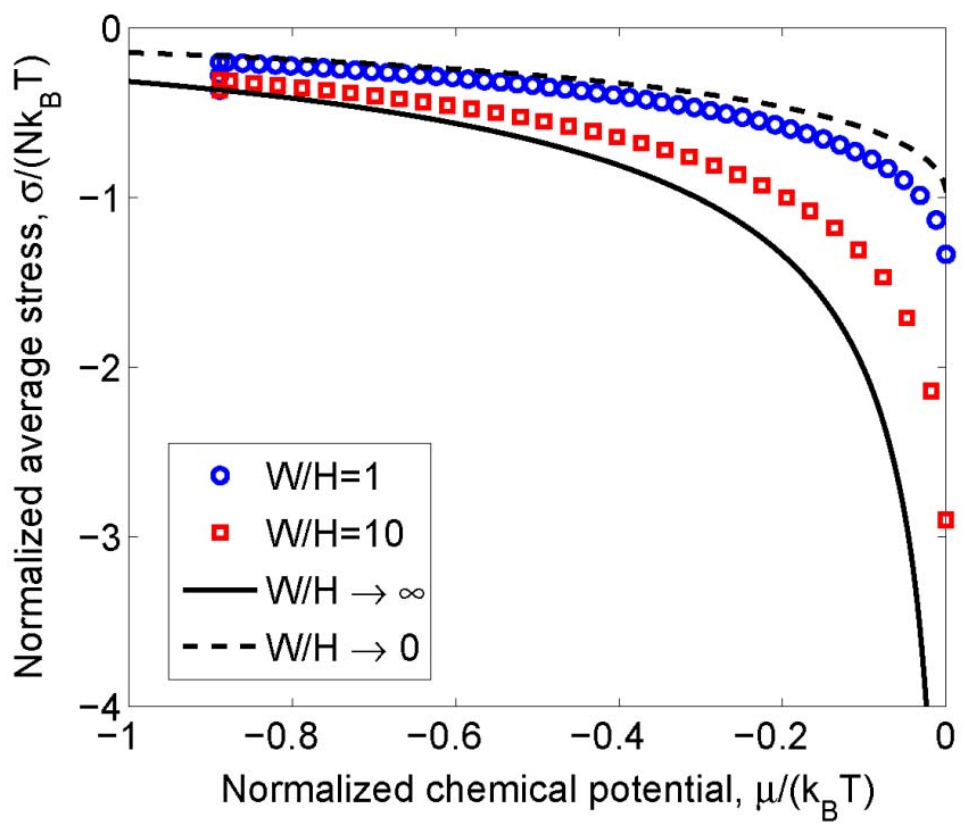

(a)

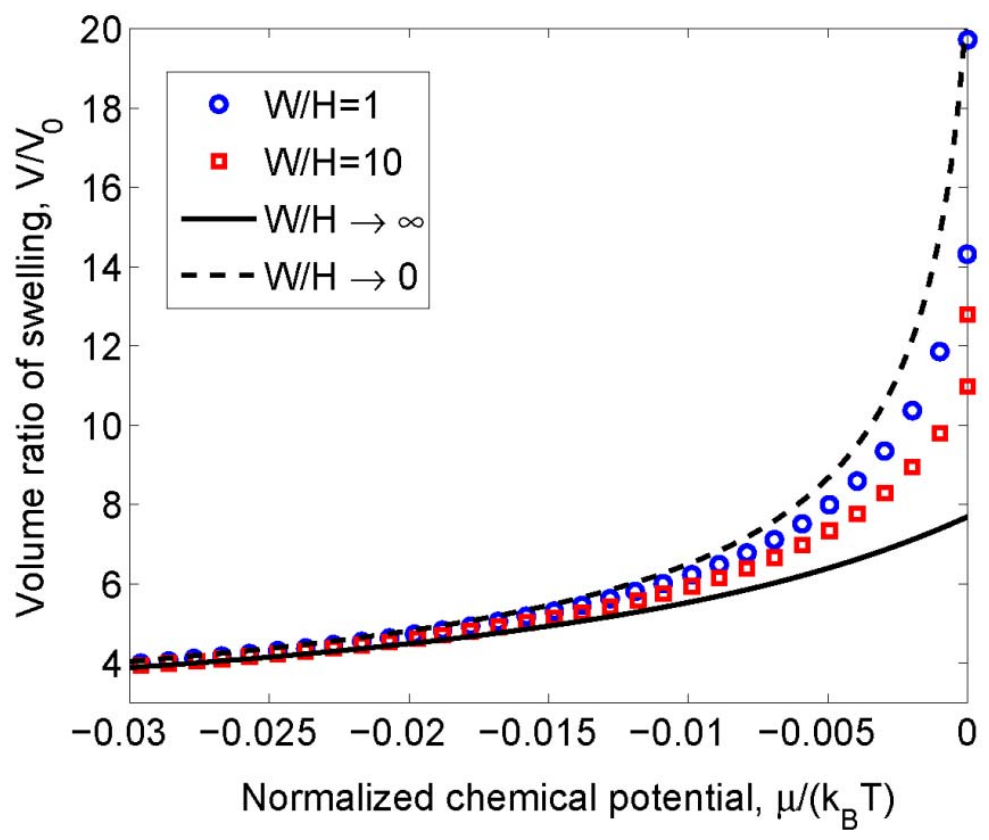

(b)

Figure 6. Inhomogeneous swelling of surface-attached hydrogel lines: (a) average longitudinal stress; (b) volume ratio of swelling. The solid and dashed lines are analytical solutions for the homogeneous limits with $W / H \rightarrow \infty$ and $W / H \rightarrow 0$, respectively. 


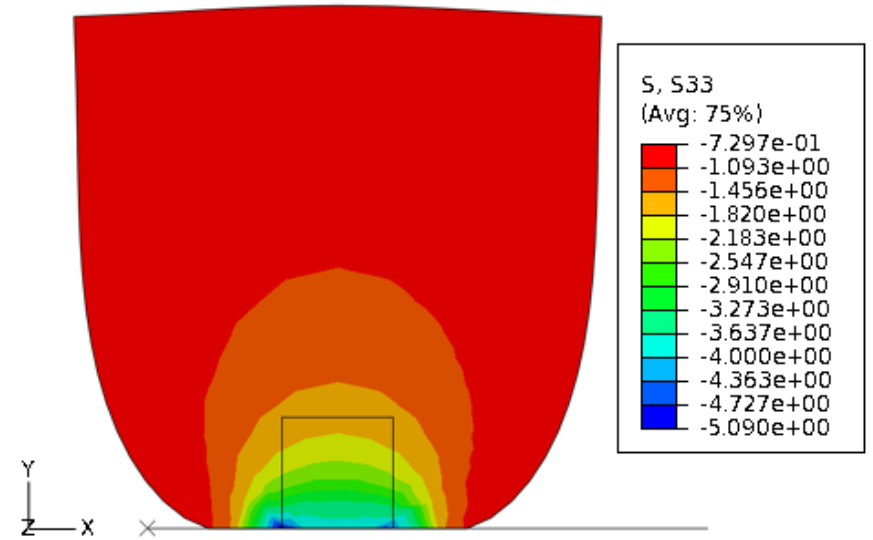

(a)

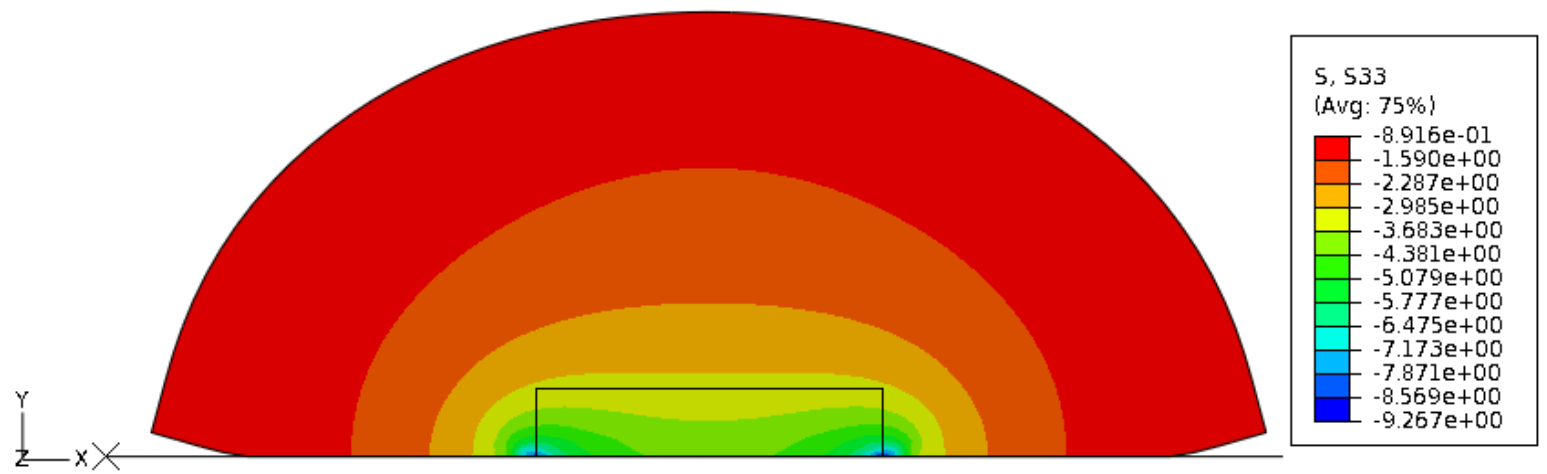

(b)

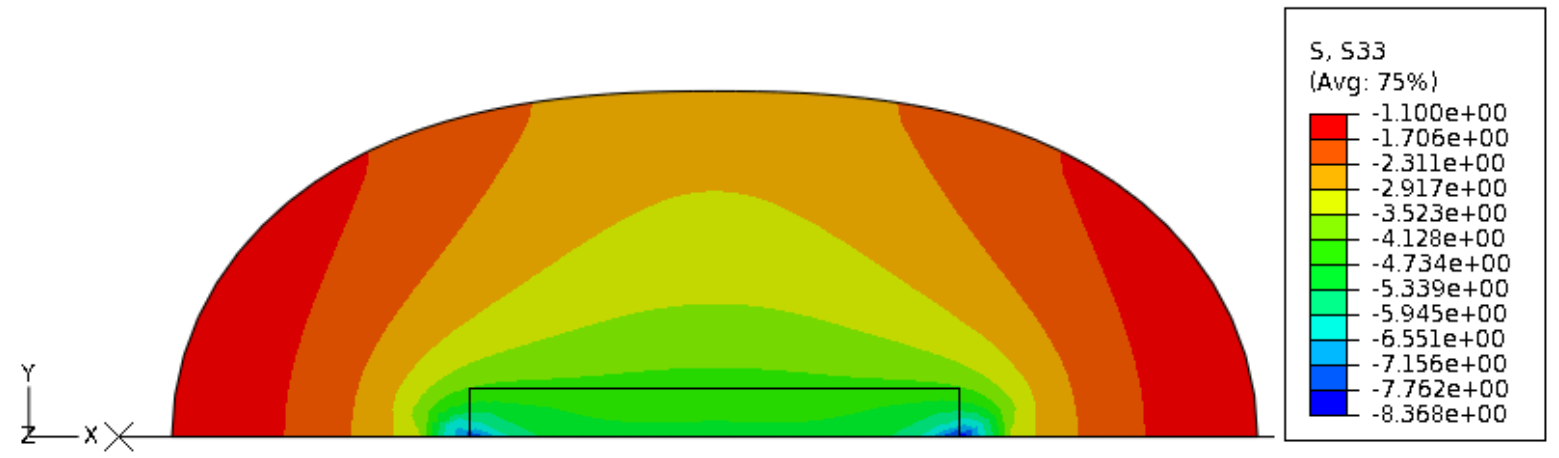

(c)

Figure 7. Simulated swelling deformation and longitudinal stress distribution in surface-attached hydrogel lines of different aspect ratios: (a) $W / H=1$; (b) $W / H=5$; (c) $W / H=10$. The rectangular boxes outline the cross sections at the dry state. 


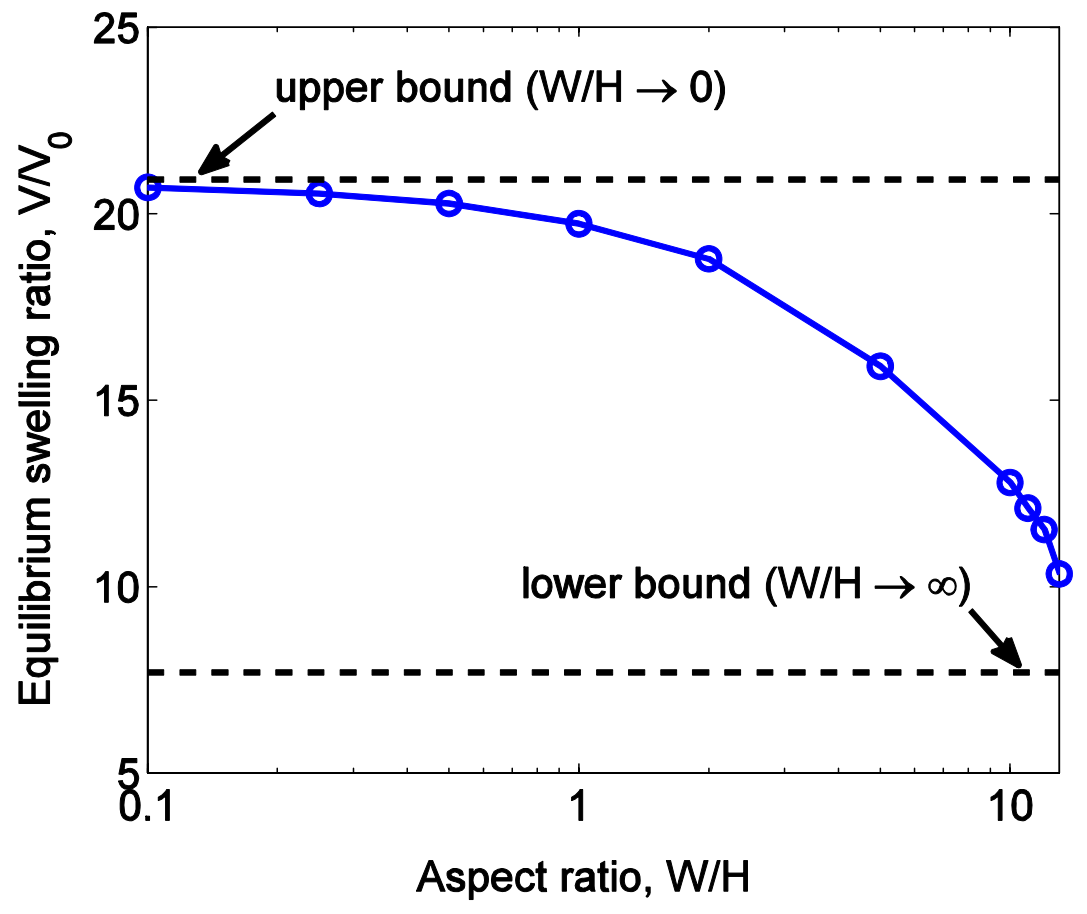

Figure 8. Equilibrium volume ratio as a function of the dry-state width-to-height aspect ratio for inhomogeneous swelling of surface-attached hydrogel lines. 


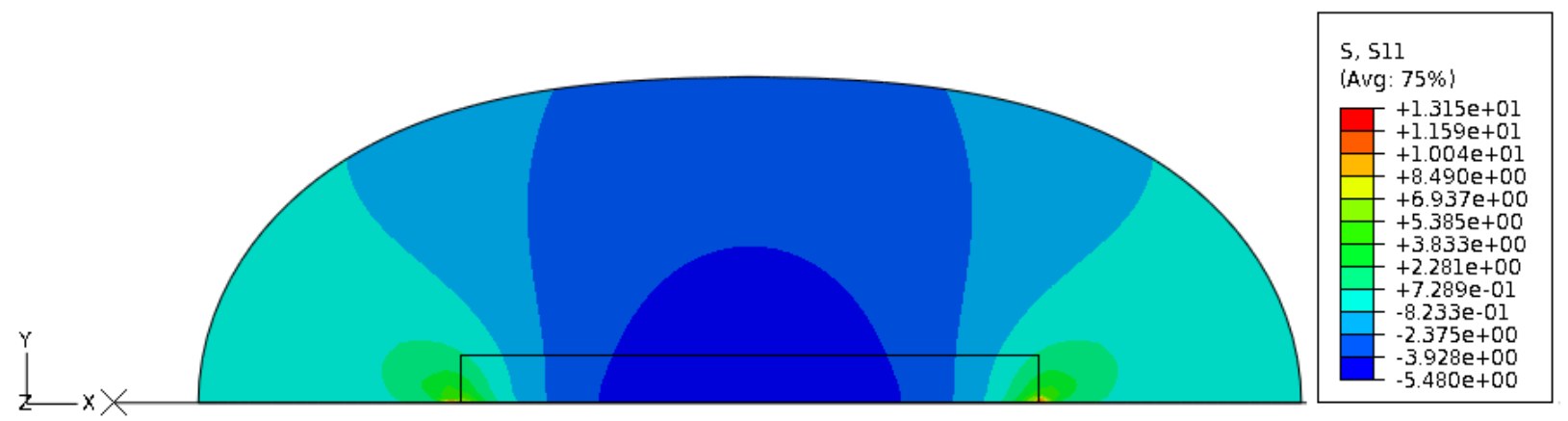

(a)

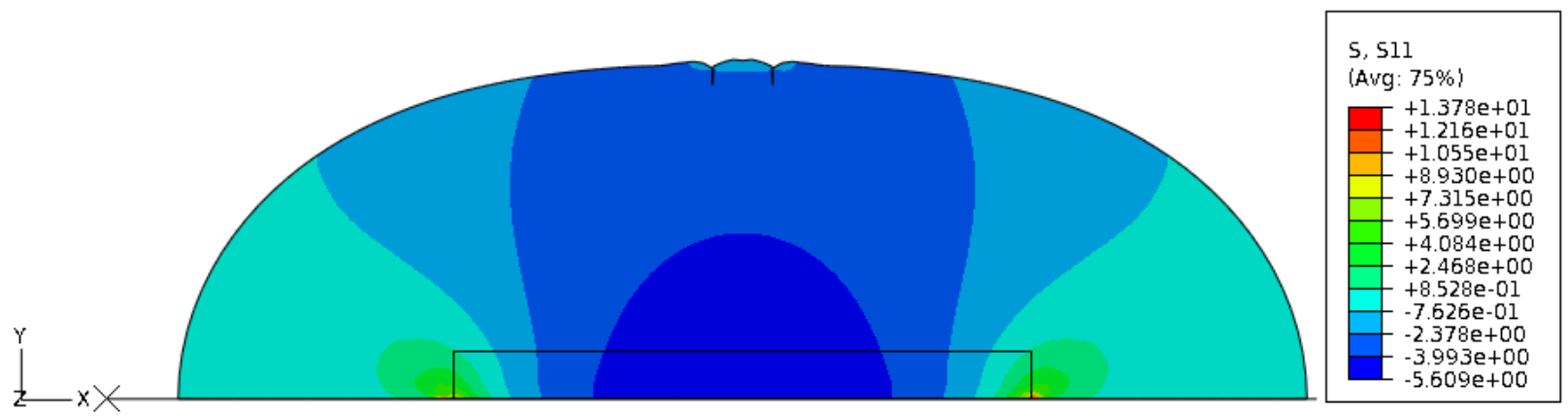

(b)

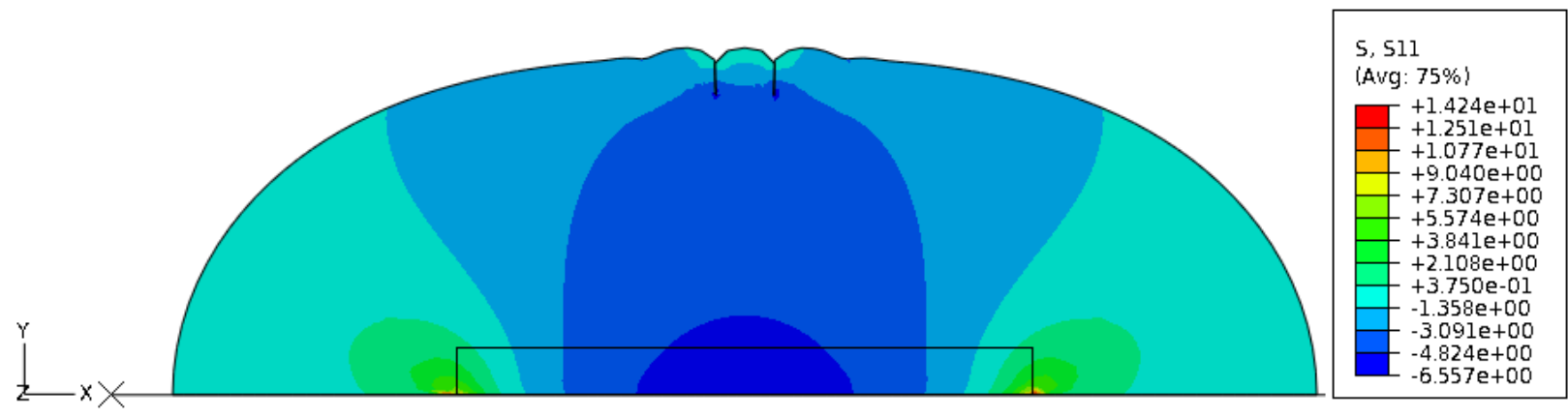

(c)

Figure 9. Formation of surface creases in a surface-attached hydrogel line with $W / H=12$ as the chemical potential increases: (a) $\bar{\mu}=-0.00075$, (b) $\bar{\mu}=-0.0003$, and (c) $\bar{\mu}=0$. 